\title{
INDUCIBLE PERIODIC HOMEOMORPHISMS OF TREE-LIKE CONTINUA ${ }^{1}$
}

BY

\author{
JUAN A. TOLEDO ${ }^{2}$
}

\begin{abstract}
In this paper we prove that every periodic homeomorphism on a tree-like continuum can be strongly induced on an inverse sequence composed of a certain kind of graph that we call "bellows". We introduce the concepts of "\#-graph" of a periodic homeomorphism and of "perfect" homeomorphism. A theorem concerning the parallel inducing of two periodic homeomorphisms having orbit spaces with the same multiplicity structure is also proved. The results are related to conjugacy and to the pseudo-arc.
\end{abstract}

1. Introduction. In this paper we are concerned with the problem of inducing periodic homeomorphisms defined on tree-like continua as inverse limits of homeomorphisms of graphs.

Many interesting examples of such homeomorphisms have been found in the past few years [Br, Lew, T] and most of them have been described as inverse limits. Certainly, this kind of description is very useful in the study of their properties and it results that it is highly desirable to be able to obtain such descriptions in terms of "nice" inverse sequences composed of "nice" spaces.

The purpose of this paper is to establish some criteria to determine when a situation is "nice" and to produce some results along those lines. The search will be oriented towards the study of conjugacy classes of periodic homeomorphisms on tree-like continua and in particular on the pseudo-arc (see [B]).

$\S \S 2$ and 3 are devoted to developing the machinery to be used in $\S \S 4-6$. This machinery is an elaboration of the techniques used by Fugate and McLean [FM].

In $\$ 4$ we introduce the concept of a "spread cover" of a continuum $M$ with respect to a periodic homeomorphism $h$. Covers of this type are finite open covers of $M$, invariant under $h$ and with the property that its elements intersect each other in a convenient way. Theorem 4.13 guarantees the existence of spread covers of arbitrarily small mesh. This allows us to construct, in the case when the orbit space is tree-like, one-dimensional invariant covers having "bellows" (see Definition 2.13) as nerves.

Received by the editors December 13, 1982.

1980 Mathematics Subject Classification. Primary 54F15, 54F20, 54F50, 54H15.

Key words and phrases.Tree-like continuum, periodic homeomorphism, inducible homeomorphism, perfect homeomorphism, conjugacy, pseudo-arc.

${ }^{1}$ This paper is part of the author's doctoral dissertation at the University of Florida under the supervision of Professor Beverly Brechner.

${ }^{2}$ During the preparation of this paper the author was supported in part by a grant from CONACYT, Mexico. 
Using a theorem by Kaul [K], we prove in Theorem 5.3 that every periodic homeomorphism whose orbit space is tree-like can be induced as an inverse limit of homeomorphisms of bellows. Later in $\$ 5$, motivated by a question by B. Brechner (Question 5.19), we discuss other forms of inducibility and as a result, the notion of "perfect" homeomorphism is introduced.

$\$ 6$ is more oriented to the study of conjugacy through a result concerning parallel inducibility of two periodic homeomorphisms on tree-like continua with the same orbit space (Theorem 6.3).

The relationship between the pseudo-arc and the concepts introduced is closely analyze $\otimes$ in $\$ \S 5$ and 6 , where several questions of interest are posed. Producing answers to these questions would represent an enormous contribution to the study of periodic homeomorphisms on the pseudo-arc.

The author wishes to express his gratitude to Professor Beverly Brechner, who supervised his doctoral research.

2. Preliminaries. All spaces are topological metric spaces. By a continuum we mean a connected, compact metric space.

DEFINITION 2.1. An open cover $\mathcal{Q}$ of a metric space $X$ is a collection $\mathscr{U}=\left\{U_{i}\right\}$ of open sets of $X$ such that $X \subset \cup\left\{U_{i}\right\}$. All covers are always understood as minimal, i.e., each element of the cover contains a point of $X$ not contained in any other element of the cover. If $V$ is a subset of $X$ and $\mathcal{Q}$ is a cover, $V<\mathcal{Q}$ means that $V$ is contained in some element of $\mathcal{Q}$ and is read " $V$ refines $\mathscr{Q}$ ". Similarly, if $\mathcal{V}$ is a cover, $\mathfrak{T}<\mathscr{Q}$, "W refines $\mathcal{Q}$ ", means that $V<\mathcal{Q}$ for every $V \in \mathcal{T}$. We will denote by $\mathcal{T}(U)$ the nerve of $U$.

DEFINITION 2.2. A continuum is called tree-like (chainable) iff it has covers of arbitrarily small mesh whose nerves are trees (arcs).

Definition 2.3. If $\Gamma$ is a graph, vrt $\Gamma$ and edg $\Gamma$ will denote the sets of vertices and edges of $\Gamma$, respectively. The edge between $v_{1}$ and $v_{2} \in \operatorname{vrt} \Gamma$ is denoted by $\left(v_{1}, v_{2}\right) \in \operatorname{edg} \Gamma$. Then $\left(v_{1}, v_{2}\right)$ refers to the set $\left\{v_{1}, v_{2}\right\}$ rather than to the ordered pair (see Remark 2.4). A graph without cycles is a tree whereas a tree without points of order three or above is a chain. All graphs are finite. As topological spaces, graphs are finite one-dimensional simplical complexes.

REMARK 2.4. When defining a graph $\Gamma$ we will often specify edg $\Gamma$ by saying $\left(x_{1}, x_{2}\right) \in \operatorname{edg} \Gamma$ iff $P\left(x_{1}, x_{2}\right)$, where $P\left(x_{1}, x_{2}\right)$ is a certain property of the ordered pair $\left(x_{1}, x_{2}\right)$. What we really mean is $\left(x_{1}, x_{2}\right) \in \operatorname{edg} \Gamma$ iff $P\left(x_{1}, x_{2}\right)$ or $P\left(x_{2}, x_{1}\right)$.

REMARK 2.5. If $K$ is a finite partially ordered set under $\alpha$, it can be made into a graph by defining vrt $K=K$ and $\left(x_{1}, x_{2}\right) \in \operatorname{edg} K$ iff $x_{1} \neq x_{2}, x_{1} \alpha x_{2}$ and $x_{1} \alpha x \alpha$ $x_{2}$ implies $x=x_{1}$ or $x=x_{2}$.

Definition 2.6. The set of positive integers is denoted by $N^{+}$. A set $\{n ; m\} \subset N^{+}$ is called divisible iff either $n \mid m$ or $m \mid n$.

DEFINITION 2.7. A \#-graph ("number graph") is a pair $(\Gamma, \sigma)$ where $\Gamma$ is a graph and $\sigma$ is a function $\sigma: \Gamma \rightarrow N^{+}$. If $\Gamma$ is a tree or a chain, it is called a $\#$-tree or a \#-chain (see Figure 2.1). Very often, we will refer only to $\Gamma$ whenever $\sigma$ is understood. 


$$
\begin{aligned}
& \Gamma: a^{\prime}>_{c}^{d} d-\bullet \\
& \sigma: \quad \begin{array}{l}
g \rightarrow 2 \\
b \rightarrow 1 \\
c \rightarrow 4 \\
d \mapsto 3 \\
a \rightarrow 2
\end{array} \\
& \text { K : } 2_{4}^{\prime 1} v^{\prime} 3-2
\end{aligned}
$$

The $*$-graph $k=(\Gamma, \sigma)$

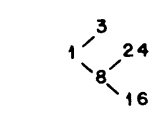

A unitary -graph $\Gamma$

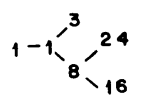

And $1+\Gamma$

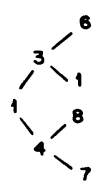

A -tree

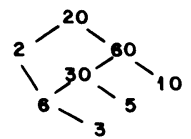

A divisible*-graph

$$
1-4-3-8
$$

$A$ - chain
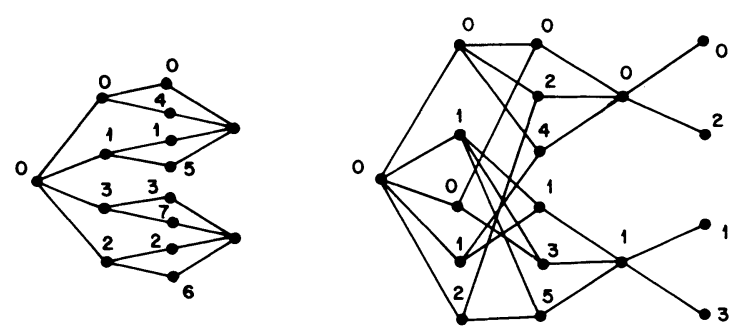

$K: 1>_{3}^{2}-6-2-4$

K-bellows

Figure 2.1

Definition 2.8. A \#-graph $(\Gamma, \sigma)$ is divisible iff whenever $(a, b) \in \operatorname{edg} \Gamma$, the set $\{\sigma(a), \sigma(b)\}$ is divisible. In this case, $\Gamma$ can be partially ordered by $x_{1} \alpha x_{2}$ iff $\left(x_{1}, x_{2}\right) \in \operatorname{edg} \Gamma$ and $\sigma\left(x_{1}\right) \mid \sigma\left(x_{2}\right)$ or $x_{1}<y_{1}<y_{2}<\cdots<y_{k}<x_{2}$ where $\left(x_{1}, y_{1}\right)$, $\left(y_{i}, y_{i+1}\right),\left(y_{k}, x_{2}\right)$ are edges of $\Gamma$ and $\sigma\left(x_{1}\right)\left|\sigma\left(y_{1}\right)\right| \sigma\left(y_{2}\right)|\cdots| \sigma\left(y_{k}\right) \mid \sigma\left(x_{2}\right)$. A divisible \#-graph is unitary if $\sigma\left(x_{0}\right)=1$ where $x_{0}$ is unique minimal under the partial ordering. In this case $1+\Gamma$ denotes the \#-graph such that $\operatorname{vrt}(1+\Gamma)=\Gamma$ $\cup\{*\}, \sigma(*)=1$ and $(*, x) \in \operatorname{edg}(1+\Gamma)$ iff $x=x_{0}$ (see Figure 2.1).

DEFINITION 2.9. Let $M$ be a continuum. A homeomorphism $h: M \rightarrow M$ is periodic iff there exists an integer $n>0$ such that $h^{n}(x)=x$ for all $x \in M$. The period of $h$ is the minimum integer $n$ with the property. Assume now that $h$ is periodic. If $x \in M$, the order of $x$, ord $x$, is the minimum positive integer $m$ such that $h^{m}(x)=x$. The orbit of $x$ is $O(x)=\left\{h(x), h^{2}(x), \ldots, h^{\text {ord } x}(x)\right\}$. The set of orbits of points of $M$ forms a partition of $M$ and when given the quotient topology becomes the orbit space of $M$ under $h$, denoted $M_{h}$. Denote by $\pi: M \rightarrow M_{h}$ the projection map. If $x \in M_{h}$, the multiplicity of $x$, mult $x$, is the number of elements of the orbit represented by $x$; that is, the cardinality of $\pi^{-1}(x)$.

LEMMA 2.10. If $h$ is periodic then $\pi$ is open and closed, and if $\left\{U_{i}\right\}$ is a finite family of subsets of $M_{h}$ then $\cap\left\{U_{i}\right\} \neq \varnothing$ iff $\cap\left\{\pi^{-1}\left(U_{i}\right)\right\} \neq \varnothing$.

Proof. Let $h$ have period $n$ and let $U$ be an open (resp. closed) set of $M$. Then $\pi^{-1} \pi(U)=\bigcup\left\{h^{i}(U) \mid i=0, \ldots, n-1\right\}$ which is open (resp. closed) since $h^{i}(U)$ is open (resp. closed). Then $\pi(U)$ is open (resp. closed). Sufficiency of the second 
statement follows from $\pi\left(\cap\left\{\pi^{-1}\left(U_{i}\right)\right\}\right) \subset \cap\left\{U_{i}\right\}$. For necessity, let $x \in \cap\left\{U_{i}\right\}$. Then there exists, for every $i$, an $x_{i} \in \pi^{-1}\left(U_{i}\right)$ such that $\pi\left(x_{i}\right)=x$. But then $O\left(x_{i}\right) \subset \pi^{-1}\left(U_{i}\right)$ for all $i$ and since $O\left(x_{i}\right)=O\left(x_{j}\right), \cap\left\{\pi^{-1}\left(U_{i}\right)\right\} \neq \varnothing$.

DeFINITION 2.11. Let $h: M \rightarrow M$ be a periodic homeomorphism on a continuum $M$. Let $\Gamma_{h}=\left\{i \in N^{+} \mid i=\right.$ ord $x$ for some $\left.x \in M\right\}$. For each $i \in \Gamma_{h}$ let $F_{i}=$ $\{x \in M \mid$ ord $x \mid i\}$. If $M$ is tree-like, each $F_{i}$ is a nonempty continuum [FM]. Furthermore, $F_{i} \subset F_{j}$ iff $i \mid j$ and $\cup\left\{F_{i} \mid i \in \Gamma_{h}\right\}=M$. Hence $\Gamma_{h}$ and $\left\{F_{i}\right\}$, partially ordered by divisibility and containment, respectively, can be thought of as isomorphic graphs (see Remark 2.5). The \#-graph of $h$, denoted also by $\Gamma_{h}$, is the pair $\left(\Gamma_{h}, \sigma\right)$ where $\sigma(i)=i$. A \#-graph $\Gamma$ is admissible iff $\Gamma=\Gamma_{h}$ for some $h$ periodic on some tree-like continuum $M$. The homeomorphism $h$ is divisible iff its \#-graph is a chain. In this case $\Gamma_{h}=\left\{n_{1}<n_{2}<\cdots<n_{p}\right\}$ and $F_{n_{1}} \subset F_{n_{2}} \subset \cdots \subset F_{n_{p}}=M$. If $M$ is tree-like then $\Gamma_{h}$ is unitary.

LEMMA 2.12. Let $h$ be a periodic homeomorphism on a tree-like continuum $M$. Then $\Gamma_{h}$ is divisible (as a \#-graph) and connected (as a topological space).

Proof. That $\Gamma_{h}$ is divisible derives directly from its definition.

Suppose $\Gamma_{h}$ is not connected. Then $\Gamma_{h}=A_{1} \cup A_{2}$ where $A_{1}$ and $A_{2}$ are subgraphs of $\Gamma_{h}$ such that no edge of $\Gamma_{h}$ connects them. Let $M_{i}=\cup\left\{F_{j} \mid j\right.$ is a maximal element of $A_{i}$ under the partial ordering (see Definition 2.8). Then $M_{\mathrm{i}}$ and $M_{2}$ are closed and $M=M_{1} \cup M_{2}$. If $x \in M_{1} \cap M_{2}$ with ord $x=k$, then there are $j_{1} \in A_{1}$ and $j_{2} \in A_{2}$ minimal with the property that $k \mid j_{1}$ and $k \mid j_{2}$. Thus $\left(k, j_{1}\right)$ and $\left(k, j_{2}\right)$ are in edg $\Gamma_{h}$, so $A_{1}$ and $A_{2}$ are connected by an edge, a contradiction. Thus, $M_{1} \cap M_{2}=\varnothing$ so $M$ is not connected, again a contradiction.

Definition 2.13. Let $(K, \sigma)$ be a divisible \#-graph. A graph $\Gamma$ is said to be a ( $K, \sigma)$-bellows or simply a $K$-bellows iff

(i) vrt $\Gamma=\cup\left\{V_{k} \mid k \in \operatorname{vrt} K\right\}$ where $V_{k}=\left\{v_{k 1}, \ldots, v_{k \sigma(k)}\right\}$ and

(ii) $\left(v_{k i}, v_{l j}\right) \in \operatorname{edg} \Gamma$ iff

(a) $(k, l) \in \operatorname{edg} K$,

(b) $i \equiv j \bmod (\min \{\sigma(l), \sigma(k)\})$ (see Figure 2.1).

REMARK 2.14. It is not hard to prove that for a fixed divisible $\#$-graph $K$, any two $K$-bellows are isomorphic. Thus we can talk of the $K$-bellows.

Definition 2.15. Let $(K, \sigma)$ be a divisible \#-chain and let $k, l, m \in \operatorname{vrt} K$ be such that $(k, l),(l, m) \in \operatorname{edg} K$. We say that the triple $k-l-m$ is a redundancy iff either $\sigma(k)<\sigma(l)=\sigma(m)$ or $\sigma(k)=\sigma(l)>\sigma(m)$. The divisible \#-chain $\left(K^{\prime}, \sigma\right)$ obtained from $(K, \sigma)$ by eliminating the redundancy $k-l-m$ is the \#-chain such that $\operatorname{vrt} K^{\prime}=\operatorname{vrt} K-\{l\}$, edg $K^{\prime}=(\operatorname{edg} K-\{(k, l),(l, m)\}) \cup\{(k, m)\}$ and $\sigma$ is the same function restricted. A divisible \#-chain is reduced iff it does not contain any redundancy.

LEMMA 2.16. For any divisible \#-chain $\Gamma$ there exists a unique reduced \#-chain that can be obtained from $\Gamma$ by eliminating a finite number of redundancies.

Proof. Given any divisible \#-chain, it contains only a finite number of redundancies. In whatever order they are eliminated, the same \#-chain is obtained and this is reduced. 
DEFINITION 2.17. The reduced \#-chain obtained in Lemma 2.16 is called the reduced -chain of $\Gamma$ and it is denoted by $\tilde{\Gamma}$.

LEMMA 2.18. If $\tilde{\Gamma}$ is the reduced \#-chain of $\Gamma$, then the $\Gamma$-bellows and the $\tilde{\Gamma}$-bellows are homeomorphic.

Proof. Let $\Gamma=(K, \sigma)$. It is enough to prove that the $\Gamma$-bellows $\Phi$ and the $\Gamma^{\prime}$-bellows $\Phi^{\prime}$ are homeomorphic, where $\Gamma^{\prime}$ is obtained from $\Gamma$ by eliminating one redundancy $k-l-m$, where $\sigma(k)<\sigma(l)=\sigma(m)$.

There are exactly $\sigma(m)$ edges in $\Phi^{\prime}$ going from $V_{k}$ to $V_{m}$, namely, $\left\{\left(v_{k i}, v_{m j}\right) \mid i=\right.$ $1, \ldots, \sigma(m) ; i \equiv j \bmod \sigma(k)\}$. Select a point $V_{l^{\prime} i}$ in the interior of each of the edges $\left(v_{k i}, v_{m j}\right)$. Define a function $\psi: \operatorname{vrt} \Phi \rightarrow \operatorname{vrt} \Phi^{\prime} \cup\left\{v_{l^{\prime} i}\right\}$ by $\psi\left(v_{x i}\right)=v_{x i}$ if $x \neq l$ and $\psi\left(v_{l i}\right)=v_{l^{\prime} i}$. Extend this one-to-one correspondence linearly to the whole set $\Phi$ to obtain the desired homeomorphism.

Lemma 2.19. Let $(K, \sigma)$ be a divisible \#-chain such that $\sigma$ is monotone under the total order of $K$. Then the $(K, \sigma)$-bellows is a tree.

Proof. Use induction on the number of vertices of $K$. If $K$ has one vertex, the lemma is trivial.

Suppose that $K=\left\{v_{1}<v_{2}<\cdots<v_{n}\right\}$. Then $\left.\sigma\right|_{K-\left\{v_{n}\right\}}$ is also monotone so, by the induction hypothesis, the $\left(K-\left\{v_{n}\right\},\left.\sigma\right|_{K-\left\{v_{n}\right\}}\right)$-bellows is a tree. Since $\sigma\left(v_{n-1}\right) \mid$ $\sigma\left(v_{n}\right)$, for each $i$ such that $0 \leqslant i<\sigma\left(v_{n}\right)$ there exists exactly one integer $j, 0 \leqslant j<$ $\sigma\left(v_{n-1}\right)$, such that $i \equiv j \bmod \sigma\left(v_{n-1}\right)$. Therefore, out of every vertex generated by $v_{n}$ there is exactly one edge. Then the $(K, \sigma)$-bellows is a tree.

LEMMA 2.20. Let $(\Gamma, \sigma)$ be a \#-chain. Then $(\Gamma, \sigma)$ is admissible iff $\Gamma$ is connected, unitary and divisible and $\sigma$ is monotone.

Proof. By Lemma 2.12, one way is clear. So let us suppose that $\Gamma$ is connected, unitary and divisible and $\sigma$ is monotone.

Consider the $(\Gamma, \sigma)$-bellows $K$. Define a function $h: \operatorname{vrt} K \rightarrow \operatorname{vrt} K$ by $h\left(v_{x i}\right)=$ $v_{x(i+1 \bmod \sigma(x))}, x \in \Gamma$. The function $h$ is a permutation on vrt $K$ that extends by linearity to a homeomorphism $h: K \rightarrow K$. Let $n$ be the maximal element of $\sigma(\Gamma)$. Then $h$ is periodic of period $n$.

By Lemma 2.19 and since $\Gamma$ is unitary and connected, $K$ is a tree-like continuum.

Now consider $\Gamma_{h}$. In order to prove that $\Gamma=\Gamma_{h}$, it is sufficient to prove that $\Gamma_{h}=\{i \mid i=\operatorname{ord} x$ for some $x \in K\}$ equals $\sigma(\Gamma)$. If $i \in \sigma(\Gamma)$, then $\sigma(x)=i$ for some $x \in \operatorname{vrt} \Gamma$. The point $v_{x 0}$ has order $\sigma(x)=i$ so $i \in \Gamma_{h}$. Conversely, if $i \in \Gamma_{h}$, then ord $y=i$ for some $y \in K$. If $y \in \operatorname{vrt} K$ then $y=v_{x j}$ for some $x \in \Gamma$ and then $\sigma(x)=i$, so $i \in \sigma(\Gamma)$. If $y \notin \operatorname{vrt} K$, then the whole edge containing it has order $i$. Thus one vertex of it must also have order $i$. Thus $\Gamma_{h}=\sigma(\Gamma)$.

REMARK 2.21. The homeomorphism described in the proof above constitutes an example of a perfect homeomorphism as defined in Definition 5.7.

3. Lifts. Throughout this section $h: M \rightarrow M$ will denote a periodic homeomorphism of a continuum $M$ onto itself, $M_{h}$ its orbit space and $\pi: M \rightarrow M_{h}$ the projection map. 

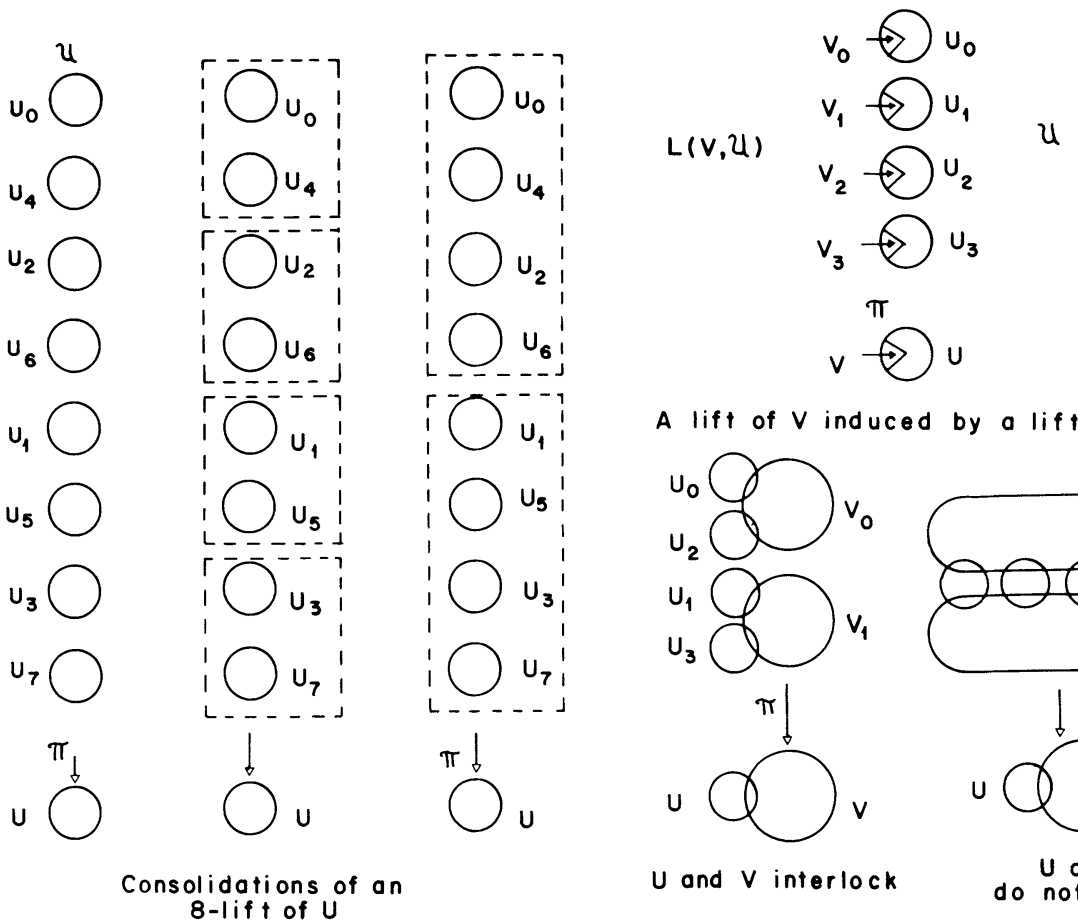

A lift of $V$ induced by a lift of $U$
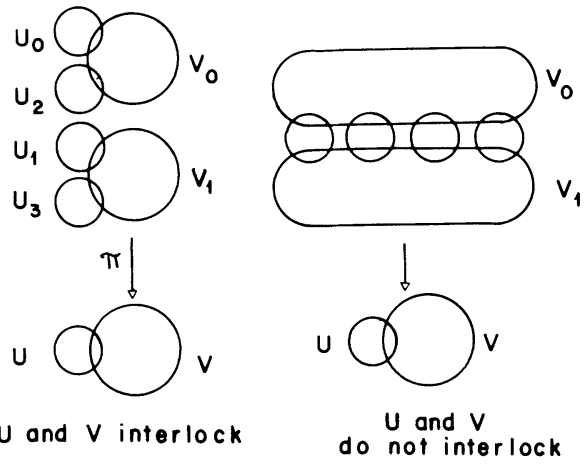

FIGURE 3.1

Definition 3.1. Let $U$ be an open set in $M_{h}$. A lift of order $n$ or an $n$-lift of $U$ is a collection of open sets of $M, \mathscr{Q}=\left\{U_{i} \mid i=0, \ldots, n-1\right\}$ such that

(i) $U_{i} \cap U_{j}=\varnothing$ iff $i \neq j$,

(ii) $h^{s}\left(U_{i}\right)=U_{i+s \bmod n}$,

(iii) $\pi^{-1}(U)=\bigcup\left\{U_{i} \mid i=0, \ldots, n-1\right\}$.

We write ord $\mathcal{U}=n$ (see Figure 3.1).

REMARK 3.2. The labeling of the elements only specifies the way they are circularly ordered. It is therefore immaterial from what element the labeling starts. On many occasions we will change the starting point if it is more convenient.

There may be several different lifts of the same order for a fixed open set $U$ as it is illustrated in the following

EXAmple 3.3. Let $U=Z \cup W$ where $Z$ and $W$ are open disjoint sets of $M_{h}$. Suppose that $Z$ and $W$ have 2-lifts $\left\{Z_{1}, Z_{2}\right\}$ and $\left\{W_{1}, W_{2}\right\}$. Then $\left\{Z_{1} \cup W_{1}, Z_{2} \cup\right.$ $\left.W_{2}\right\}$ and $\left\{Z_{1} \cup W_{2}, Z_{2} \cup W_{1}\right\}$ are two different 2-lifts of $U$.

Definition 3.4. Let $\mathcal{Q}=\left\{U_{i} \mid i=0, \ldots, n-1\right\}$ be an $n$-lift of $U \subset M_{h}$. Let $m \mid n$. The $m$-consolidation of $\mathscr{U}$, denoted $\mathcal{U}_{m}$, is defined by $\mathcal{U}_{m}=\left\{V_{i} \mid i=0, \ldots, m-1\right\}$ where $V_{i}=\bigcup\left\{U_{k} \mid k \equiv i \bmod m\right\}$.

LEMMA 3.5. If $थ$ is an n-lift of $U$ and $m \mid n$, then $\mathscr{U}_{m}$ is an m-lift of $U$.

Proof. (i) If $V_{i} \cap V_{j} \neq \varnothing$ for $0 \leqslant i, j \leqslant m-1$, then, since the $U_{i}$ 's are pairwise disjoint, $U_{k} \subset V_{i} \cap V_{j}$ for some $k$ such that $k \equiv i \bmod m$ and $k=j \bmod m$. But this implies $i=j$. 
(ii) Since $m \mid n, p \equiv q \bmod m$ iff $p \equiv(q \bmod n) \bmod m$.

Thus we have

$$
\begin{aligned}
h^{s}\left(V_{i}\right) & =\bigcup\left\{U_{k+s \bmod n} \mid k \equiv i \bmod m\right\}=\bigcup\left\{U_{l} \mid l \equiv i+s \bmod m\right\} \\
& =\bigcup\left\{U_{l} \mid l \equiv(i+s \bmod n) \bmod m\right\}=V_{i+s \bmod n}
\end{aligned}
$$

(iii) Clear.

LEMMA 3.6. If $\mathscr{Q}$ is an $n$-lift of $U$ and $l|m| n$ then $\mathscr{U}_{m}<\vartheta_{i^{\prime}}$.

Proof. Let $\mathscr{U}_{m}=\left\{W_{i} \mid i=0, \ldots, m-1\right\}$ and $\mathscr{U}_{l}=\left\{V_{i} \mid i=0, \ldots, l-1\right\}$. If $U_{k}$ $\subset W_{i}$, then $k \equiv i \bmod m$ so $k \equiv i \bmod l$ and thus $k \equiv(i \bmod l) \bmod l$. From here we conclude that $U_{k} \subset V_{i_{\bmod / l}}$ so $W_{i} \subset V_{i \bmod /}$ (see Figure 3.1).

LEMMA 3.7. If $\mathscr{Q}$ is an $m$-lift of $U$ and $l \mid m$ then $\left(\mathcal{Q}_{m}\right)_{l}=\mathscr{Q}_{l}$.

Proof. Let $\mathscr{U}_{m}=\left\{V_{i} \mid i=0, \ldots, m-1\right\}$ where $V_{i}=\bigcup\left\{U_{k} \mid k \equiv i \bmod m\right\}$. By definition, $\left(\mathcal{Q}_{m}\right)_{l}=\left\{W_{j} \mid j=1, \ldots, l\right\}$ where

$$
\begin{aligned}
W_{j} & =\bigcup\left\{V_{i} \mid i \equiv j \bmod l\right\}=\bigcup\left\{U_{k} \mid k \equiv i \bmod m, i \equiv j \bmod l\right\} \\
& =\bigcup\left\{U_{k} \mid k \equiv j \bmod l\right\} .
\end{aligned}
$$

But this is nothing more than the $j$ th element of $\mathscr{U}_{l}$.

There is an obvious connection between lifts and cyclic groups.

An $n$-lift can be identified with $Z_{n}$ where $s$ applications of $h$ correspond to adding the number $s$ modulo $n$. This way, the consolidations correspond to the quotient groups of $Z_{n}\left({ }^{Q}{ }_{m} \leftrightarrow Z_{n} / Z_{n / m}\right)$ and the last lemma derives from the fact that the set of quotients of $Z_{n}$ constitutes a lattice isomorphic to the set of divisors of $n$.

For the sake of simplicity it is preferable not to invoke this connection and include direct proofs of the results even though they resemble very much the corresponding ones for groups.

LEMma 3.8. Let $\mathscr{U}=\left\{U_{i}\right\}$ be an n-lift of $U$. Then $h^{s}\left(U_{i}\right)=U_{i}$ iff $n \mid s$.

Proof. $h^{s}\left(U_{i}\right)=U_{i+s \bmod n}$ so $i+s \bmod n=i$. This implies $s \equiv 0 \bmod n$.

Lemma 3.9. Let $\mathscr{Q}=\left\{U_{i}\right\}$ be an n-lift of $U$. If $x \in \cup\left\{U_{i}\right\}$ then $n \mid$ ord $x$. If $x \in U$ then $n \mid$ mult $x$. The same is true for $x \in \cup\left\{\bar{U}_{i}\right\}$ in the case that $\bar{U}_{i} \cap \bar{U}_{j}=\varnothing$ iff $i \neq j$.

Proof. If $x \in \cup\left\{U_{i}\right\}$ then $x \in U_{i}$ for some $i$. Apply the previous lemma to $h^{\text {ord } x}\left(U_{i}\right)=U_{i}$. If $x \in U$ then there exists $x^{\prime} \in \cup\left\{U_{i}\right\}$ such that ord $x^{\prime}=$ mult $x$. Suppose that $\bar{U}_{i} \cap \bar{U}_{j}=\varnothing$ iff $i \neq j$. Then $h^{s}\left(\bar{U}_{i}\right)=\bar{U}_{i+s \bmod n}$. Proceed using similar arguments.

Definition 3.10. Let $\mathcal{Q}=\left\{U_{i} \mid i=0, \ldots, n-1\right\}$ be an $n$-lift of $U$. If $V \subset U$ is an open set, we define $L(V, \mathcal{Q})$, the lift of $V$ induced by $\mathscr{Q}$, by $L(V, \mathcal{Q})=\left\{U_{i} \cap\right.$ $\left.\pi^{-1}(V) \mid i=0, \ldots, n-1\right\}$. If $m \mid n$ then $L_{m}(V, \mathcal{Q})$ denotes the $m$-consolidation of $L(V, \mathcal{Q})$. Since $h\left(U_{i} \cap \pi^{-1}(V)\right)=U_{i+1 \bmod n} \cap \pi^{-1}(V)$, this is well defined (see Figure 3.1).

LEMMA 3.11. Let $\mathcal{Q}$ be an $n$-lift of $U$ and $V \subset U$. For $m \mid n, L_{m}(V, \mathcal{Q})=L\left(V, \mathscr{U}_{m}\right)$. 
Proof. Both lifts are of order $m$. The $i$ th element of $L_{m}(V, \mathcal{Q})$ is

$$
\cup\left\{U_{k} \cap \pi^{-1}(V) \mid k \equiv i \bmod m\right\} \text {. }
$$

But this is equal to $\left(\cup\left\{U_{k} \mid k \equiv i \bmod m\right\}\right) \cap \pi^{-1}(V)$ which is the $i$ th element of $L\left(V, \mathfrak{Q}_{m}\right)$.

LEMMA 3.12. Let $V \subset U$ and let $\mathcal{T}$ be an $m$-lift of $V$ and $\mathscr{Q}$ an $n$-lift of $U$ such that $\mathfrak{T}<\mathscr{Q}$. Then $L(V, \mathcal{Q})=\mathcal{V}_{n}$.

Proof. Assume that $V_{0} \subset U_{0}$ (see Remark 3.2). Then $h^{m}\left(V_{0}\right)=V_{0} \subset U_{0}$ so $h^{m}\left(U_{0}\right)=U_{0}$ and then, by Lemma 3.8, $n \mid m$. Let $0 \leqslant k \leqslant m-1$ and $0 \leqslant i \leqslant n-1$. Suppose $k \equiv i \bmod n$. Then $h^{k}\left(V_{0}\right) \subset h^{k}\left(U_{0}\right)$ implies $V_{k} \subset U_{k \bmod n}$, so $V_{k} \subset U_{i}$.

Conversely, if $V_{k} \subset U_{i}$ then $h^{-k}\left(V_{k}\right) \subset h^{-k}\left(U_{i}\right)$ so $V_{0} \subset U_{i-k \bmod n}$ and, since $V_{0} \subset U_{0}, i-k \bmod n=0$ or $i \equiv k \bmod n$. Therefore $V_{k} \subset U_{i}$ iff $k \equiv i \bmod n$. Now, observe that the $i$ th element of $L(V, \mathcal{Q})$ is $\pi^{-1}(V) \cap U_{i}$. But this is exactly $\left\{V_{k} \mid k \equiv i \bmod n\right\}$ which is the $i$ th element of $\mathscr{V}_{n}$.

The necessity of the condition $\mathcal{V}<\mathcal{Q}$ can be easily illustrated as follows: Suppose

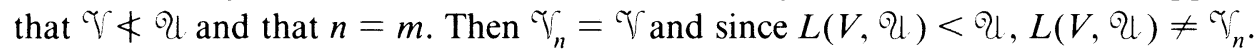

LEMmA 3.13. Let $W \subset V \subset U$ and $\mathscr{Q}$ a lift of $U$. Then $L(W, L(V, \mathcal{Q}))=L(W, \mathcal{Q})$.

Proof. Let $L(V, \mathcal{Q})=\left\{T_{i}\right\}$. The lemma follows from the fact that $\pi^{-1}(W) \cap T_{i}$ $=\pi^{-1}(W) \cap \pi^{-1}(V) \cap U_{i}=\pi^{-1}(W) \cap U_{i}$.

LEMMA 3.14. Let $W \subset V \subset U, \mathcal{V}$ an $m$-lift of $V$ and $\mathscr{Q}$ an $n$-lift of $U$ such that $\mathscr{V}<$ Q . Then

(i) $L(W$, Q $)=L_{n}(W, \mathscr{T})$.

(ii) If $p|n| m$ then $L_{p}(W, \mathscr{T})=L_{p}(W, \mathscr{Q})$.

(iii) If $n|p| m$ then $L_{p}(W, \mathscr{V})<L(W, \mathcal{Q})$.

Proof Use the three previous lemmas to prove (i) by means of $L_{n}(W, \mathscr{T})=$ $L\left(W, \mathscr{V}_{n}\right)=L(W, L(V, \mathcal{Q}))=L(W, \mathcal{Q})$.

Use Lemma 3.7 and (i) to verify (ii) as follows:

$$
L_{p}(W, \mathscr{Q})=\left(L_{n}(W, \mathscr{T})\right)_{p}=L_{p}(W, \mathscr{T})
$$

Finally, (iii) follows from Lemma 3.6 and part (i).

Standing Notation 3.15. Up to the end of the proof of Corollary 3.23 we will assume that $U, V \subset M_{h}$, Q is an $n$-lift of $U$ and $\widetilde{V}$ is an $m$-lift of $V$. Also, any time $\mathscr{Q}$ and $\mathcal{V}$ are related, we will assume (see Remark 3.2) that we have renumbered the elements of the lifts in such a way that $U_{0}$ and $V_{0}$ are also related. For example, if थ $\cap \mathcal{V} \neq \varnothing$ then $U_{0} \cap V_{0} \neq \varnothing$; if $\mathcal{Q} \subset \mathcal{V}$ then $U_{0} \subset V_{0}$, etc.

Lemma 3.16. Suppose that $U \cap V \neq \varnothing$. Then every $U_{i}$ intersects at least one $V_{j}$ and every $V_{i}$ intersects at least one $U_{j}$.

Proof. Recall from Lemma 2.10 and Standing Notation 3.15 that $U \cap V \neq \varnothing$ iff $Q \cap \mathcal{T} \neq \varnothing$ and that $U_{0} \cap V_{0} \neq \varnothing$. Then $U_{i}=h^{i}\left(U_{0}\right)$ intersects $V_{i \bmod m}$ and $V_{j}=$ $h^{j}\left(V_{0}\right)$ intersects $U_{j \bmod n}$. 
Definition 3.17. Suppose $U \cap V \neq \varnothing$. We say that $\mathscr{l}$ and $\mathscr{T}$ interlock iff

(i) the set $\{m, n\}$ is divisible,

(ii) $U_{i} \cap V_{j} \neq \varnothing$ iff $i \equiv j \bmod (\min \{n, m\})$ (see Figure 3.1).

The following lemma is a direct consequence of the definition.

LEMmA 3.18. If $W_{1} \cap W_{2} \neq \varnothing, W_{1} \subset U, W_{2} \subset V$ and $\mathcal{Q}$ and $\mathcal{T}$ interlock then $L\left(W_{1}, \mathcal{Q}\right)$ and $L\left(W_{2}, \mathfrak{T}\right)$ also interlock.

Lemma 3.19. Suppose that $\mathscr{Q}$ and $\mathscr{T}$ interlock. If $p \mid n$ and $\{p, m\}$ is divisible then $\mathrm{Q}_{p}$ and $\mathcal{T}$ interlock.

Proof. Let $\mathscr{Q}_{p}=\left\{W_{i} \mid i=0, \ldots, p-1\right\}$. By hypothesis $U_{i} \cap V_{j} \neq \varnothing$ iff $i \equiv$ $j \bmod (\min \{n, m\})$.

Case I. $p \mid m$. If $W_{i} \cap V_{j} \neq \varnothing$ then for some $U_{k} \subset W_{i}, U_{k} \cap V_{j} \neq \varnothing$. This implies that $k \equiv j \bmod (\min \{n, m\})$, so $k \equiv j \bmod p$ because $p \mid m$ and $p \mid n$. Since $U_{k} \subset W_{i}$, $k \equiv i \bmod p$ and thus $i \equiv j \bmod p$. Conversely, if $0 \leqslant i<p, 0 \leqslant j<m$ and $i \equiv$ $j \bmod p$ then $U_{j \bmod n} \subset W_{i}$ and since $(j \bmod n) \equiv j \bmod (\min \{n, m\})$ and $\mathscr{Q}$ and $\mathscr{V}$ interlock, $U_{j \bmod n} \cap V_{j} \neq \varnothing$ and so $W_{i} \cap V_{j} \neq \varnothing$.

Case II. $m \mid p$. Then $m \mid n$. Now, if $W_{i} \cap V_{j} \neq \varnothing$ then for some $U_{k} \subset W_{i}, U_{k} \cap V_{j}$ $\neq \varnothing$. This implies that $k \equiv j \bmod m$. Since $U_{k} \subset W_{i}, k \equiv i \bmod p$ and so $k \equiv$ $i \bmod m$. Therefore $i \equiv j \bmod m$. Conversely, if $0 \leqslant i<p, 0 \leqslant j<m$ and $i \equiv j \bmod m$ then, since $\mathscr{Q}$ and $\Upsilon$ interlock, $U_{i} \cap V_{j} \neq \varnothing$. Since $i \equiv i \bmod p, U_{i} \subset W_{i}$. Therefore $W_{i} \cap V_{j} \neq \varnothing$.

Lemma 3.20. Suppose $\mathcal{Q}$ and $\mathcal{T}$ interlock. If $p|n, q| m$ and $\{p, q\}$ is divisible then $\mathrm{Q}_{p}$ and $\tau_{q}$ interlock.

Proof. Suppose $p \mid q$. Then $p \mid m$ so by the previous lemma $\mathscr{Q}_{p}$ and $\widetilde{T}$ interlock. Apply the lemma again to $\mathscr{U}_{p}, \mathcal{V}_{\text {and }} q$ and conclude that $\mathscr{Q}_{p}$ and $\mathcal{V}_{q}$ interlock. The other case is similar.

COROLlaRY 3.21. Under the same hypothesis of the last lemma, if $W_{1} \subset U$ and $W_{2} \subset V$ then $L_{p}\left(W_{1}, \mathscr{Q}\right)$ and $L_{q}\left(W_{2}, \mathcal{T}\right)$ interlock.

Lemma 3.22. Suppose that $\mathscr{Q}$ and $\mathfrak{T}$ interlock and $m \mid n$. Then $L(U \cap V, \mathcal{T})=$ $L_{m}(U \cap V$, Q $)$.

Proof. This is a consequence of the fact that

$$
\begin{aligned}
\pi^{-1}(U \cap V) \cap V_{i} & =\pi^{-1}(U \cap V) \cap \cup\left\{U_{k} \mid U_{k} \cap V_{i} \neq \varnothing\right\} \\
& =\pi^{-1}(U \cap V) \cap \cup\left\{U_{k} \mid k \equiv i \bmod m\right\} \\
& =\cup\left\{\pi^{-1}(U \cap V) \cap U_{k} \mid k \equiv i \bmod m\right\} .
\end{aligned}
$$

COROLlaRY 3.23. Under the same hypothesis of the last lemma if $W \subset U \cap V$ then $L(W, \mathscr{T})=L_{m}(W, \mathscr{Q})$.

In particular, when ord $\mathscr{Q}=$ ord $\mathscr{T}$, this result shows that no matter which one of $\mathcal{U}$ and $\mathcal{T}$ we use to lift $W$, we obtain the same lift, provided $\mathscr{U}$ and $\widetilde{V}$ interlock.

Now we want to present an equivalent definition of interlocking of two lifts. 
Theorem 3.24. Suppose $\mathcal{Q} \cap \mathcal{T} \neq \varnothing$. Then, $\mathcal{Q}$ and $\mathcal{V}$ interlock iff each $U_{i}$ intersects exactly one $V_{j}$ or each $V_{i}$ intersects exactly one $U_{j}$.

Proof. Suppose that $\mathscr{Q}$ and $\mathscr{T}$ interlock and $m \mid n$. If $U_{i}$ intersects both $V_{j}$ and $V_{k}$ then $j \equiv k \bmod m$ so $V_{j}=V_{k}$, since $0 \leqslant j, k \leqslant m-1$.

Suppose now that $\mathscr{U}$ and $\mathscr{V}$ do not interlock.

If $\{m, n\}$ is not divisible, let $x \in U_{0} \cap V_{0}$. Then $h^{m}(x) \in h^{m}\left(U_{0}\right) \cap h^{m}\left(V_{0}\right)$. By Lemma 3.8, $h^{m}\left(U_{0}\right) \neq U_{0}$ and $h^{m}\left(V_{0}\right)=V_{0}$. Then $V_{0}$ intersects both $h^{m}\left(U_{0}\right)$ and $U_{0}$. Similarly $h^{n}(x) \in h^{n}\left(U_{0}\right) \cap h^{n}\left(V_{0}\right)$ so $U_{0}$ intersects both $V_{0}$ (cf. 3.15) and $h^{n}\left(V_{0}\right)$.

If $\{m, n\}$ is divisible, say $m \mid n$, and for some $i, j, U_{i} \cap V_{j} \neq \varnothing$ and $i \neq j \bmod m$, then let $x \in U_{i} \cap V_{j}$. Then $h^{m}(x) \in h^{m}\left(U_{i}\right) \cap h^{m}\left(V_{j}\right)$. By Lemma 3.8, $h^{m}\left(U_{i}\right) \neq U_{i}$ and $h^{m}\left(V_{j}\right)=V_{j}$. Then $V_{j}$ intersects both $U_{i}$ and $h^{m}\left(U_{i}\right)$. Similarly $h^{-i}(x) \in h^{-i}\left(U_{i}\right)$ $\cap h^{-i}\left(V_{j}\right)$ so $U_{0}$ intersects both $V_{0}$ (cf. 3.15) and $V_{j-i \bmod m}$.

In any case, we just proved that if $\mathscr{Q}$ and $\mathcal{V}$ do not interlock then some $U_{i}$ intersects more than one $V_{j}$ and some $V_{i}$ intersects more than one $U_{j}$.

Lemma 3.25. Let $C=\left\{C_{i} \mid i=1, \ldots, n\right\}$ be an open finite cover of $M_{h}$. Suppose that for each $C_{i} \in C$ there exists a lift $L\left(C_{i}\right)$ of order $n_{i}$ in such a way that whenever $C_{i} \cap C_{j} \neq \varnothing$ then $L\left(C_{i}\right)$ and $L\left(C_{j}\right)$ interlock. Then $\hat{C}=\cup\left\{L\left(C_{i}\right) \mid i=1, \ldots, n\right\}$ is a finite open cover of $M$ invariant under $h$. Furthermore $\operatorname{dim} \hat{C}=\operatorname{dim} C$.

Proof. Let $U \in \hat{C}$. Then $U \in L\left(C_{i}\right)$ for some $i$, which is a lift, so $h(U) \in L\left(C_{i}\right)$ $\subset \hat{C}$. Therefore $\hat{C}$ is invariant. Let $W_{j} \in \hat{C}$ for $j=1, \ldots, m$. Since all the elements of each $L\left(C_{i}\right)$ are disjoint, $\cap\left\{W_{j} \mid j=1, \ldots, m\right\} \neq \varnothing$ iff $W_{j} \in L\left(C_{i}\right)$ where all $C_{i}$,'s are distinct. But, by Lemma 2.10, this happens iff $\cap\left\{C_{i} \mid j=1, \ldots, m\right\}=\varnothing$.

LEMMA 3.26. In addition to the hypothesis of the previous lemma, suppose that $C$ is a tree-cover of $M_{h}$. Then $\mathcal{X}(\hat{C})$ is the $(C, \sigma)$-bellows, where $\sigma\left(C_{i}\right)=n_{i}$. Furthermore, $\bar{h}$ : $\Re(\hat{C}) \rightarrow \Re(\hat{C})$, the map induced by $h$, is an isomorphism which is also an isometry.

Proof. If $C$ is a tree-cover, it is certainly a graph where the vertices are the elements of $C$ and $\left(C_{1}, C_{2}\right)$ is an edge iff $C_{1} \cap C_{2} \neq \varnothing$. Define $K=(C, \sigma)$ by $\sigma\left(C_{i}\right)=$ ord $L\left(C_{i}\right)=n_{i}$. By the interlocking condition in the hypothesis, $K$ is a divisible \#-graph so it makes sense to talk about the $K$-bellows.

Let us verify that $\mathscr{N}(\hat{C})$ is indeed the $K$-bellows. Since $C$ is a tree-cover we can assume that if $i>1, C_{i}$ intersects exactly one $C_{j}$ with $j<i$. Give any labeling (cf. Remark 3.2) to the elements of $L\left(C_{1}\right)$. Label the elements of $L\left(C_{2}\right)$ in such a way that the zero-labeled elements intersect. Continue labeling the elements of $L\left(C_{i}\right)$ consistently with the labeling of the elements of the unique $L\left(C_{j}\right), j<i$, that it intersects. At the end, the zero-labeled elements of lifts that intersect will intersect.

Now recall that $\Re(\hat{C})$ is a finite simplical complex, a graph, whose set of vertices is $\cup\left\{L\left(C_{i}\right) \mid i=1, \ldots, n\right\}$ where $L\left(C_{i}\right)=\left\{U_{i 1}, U_{i 2}, \ldots, U_{i n_{i}}\right\}$. Observe next that $\left(U_{i l}, U_{j k}\right)$ is an edge of $\mathcal{X}(\hat{C})$ iff $C_{i} \cap C_{j} \neq \varnothing$ and $i \equiv j \bmod \left(\min \left\{n_{i}, n_{j}\right\}\right)$. This is true since $L\left(C_{i}\right)$ and $L\left(C_{j}\right)$ interlock. But this happens iff $\left(C_{i}, C_{j}\right)$ is an edge of $C$ and $i \equiv j \bmod \left(\min \left\{\sigma\left(C_{i}\right), \sigma\left(C_{j}\right)\right\}\right)$. Then $\mathscr{N}(\hat{C})$ is a $K$-bellows.

By the previous lemma, $\hat{C}$ is an invariant cover. The map $\bar{h}$ is defined by the linear extension of the correspondence $U_{i l} \rightarrow h\left(U_{i l}\right)$. Since $h$ is a homeomorphism, $\bar{h}$ is an 
isomorphism. By properly choosing the metric in the nerve (Kaul $[\mathbf{K}]), \bar{h}$ becomes an isometry.

The necessity of $C$ being a tree-cover is illustrated by the following

EXAMPLE 3.27. Let $M$ be the space shown in Figure 3.2(a) with $h$ being the period-2 homeomorphism that maps parts labeled with "l" to parts labeled with "2". Figure 3.2(b) shows $M_{h}$.

Consider the cover $C$ of Figure 3.2(d). $C$ is not a tree and lifts to the graph $\hat{C}$ shown in Figure 3.2(c). The number graph $(C, \sigma)$ (Figure 3.2(e)) has its bellows displayed in Figure 3.2(f), (g). But $\Re(\hat{C})$ (Figure 3.2(h)) is not the $(C, \sigma)$-bellows!

The difficulty arises in trying to label the elements of the lifts of each $C_{i}$ in a consistent way as illustrated in Figure 3.2(i).

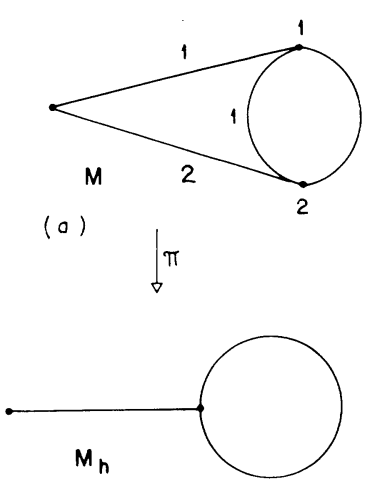

(b)

$$
1-2-2-2-2>\frac{2}{1}
$$

(e)
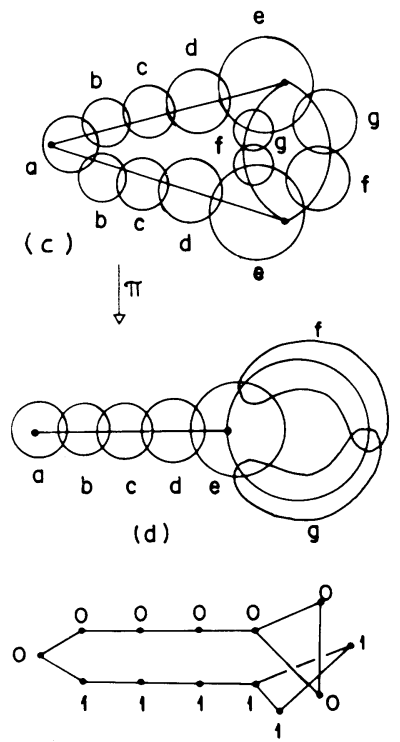

(f)
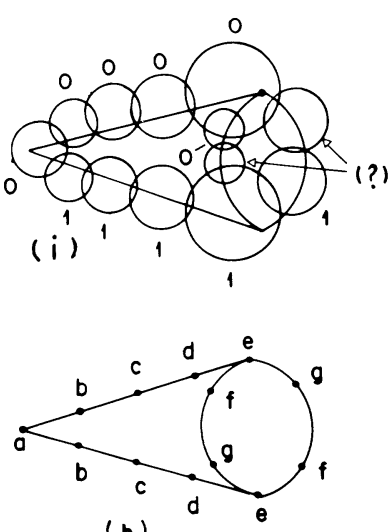

$(h)$

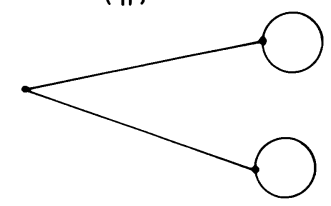

(g)

FIGURE 3.2

4. Round and spread covers. In this section we introduce the concept and prove the existence of a very useful type of invariant cover that allows us to construct invariant one-dimensional covers having bellows as nerves.

The main results of this section are Theorems 4.13, 4.17, 4.18, 4.22 and 4.24, and Corollary 4.20.

Throughout this section we assume that $h: M \rightarrow M$ is a period- $n$ homeomorphism of a continuum $M$. We will denote the orbit space of $h$ by $M_{h}$ and $\pi: M \rightarrow M_{h}$ will be the projection map.

Since $M$ is metric and the set $\left\{h, h^{2}, h^{3}, \ldots, h^{n}\right\}$ is an equicontinuous group of homeomorphisms we can endow $M$ with a metric $d: M \times M \rightarrow R$ with respect to which $h$ is an isometry [A, p. 604]. Denote by $B_{\delta}(x)$ the set $\{y \in M \mid d(x, y)<\delta\}$, 
i.e., the ball with center at $x$ and radius $\delta$. Since $h$ is an isometry $h^{s}\left(B_{\delta}(x)\right)=$ $B_{\delta}\left(h^{s}(x)\right)$. Denote this set by $B_{\delta}^{s}(x)$. Observe that if $h(x)=x$ then $B_{\delta}^{s}(x)=B_{\delta}^{t}(x)$ for all $s$ and $t$.

Lemma 4.1. For every $x \in M$ there exists a $\gamma>0$ such that the set $\left\{\overline{B_{\delta}^{s}(x)} \mid s=\right.$ $0, \ldots$, ord $x-1\}$ is pairwise disjoint.

Proof. Let $\gamma<\alpha / 2$ where

$$
\alpha=\min \left\{d\left(h^{s}(x), h^{t}(x)\right) \mid 0 \leqslant s \neq t \leqslant \text { ord } x-1\right\} .
$$

DEFINITION 4.2. A number $\gamma$ with the above property is called a splitting number for $x$.

Observe that if $\gamma$ is a splitting number for $x$ so is any $\delta$ such that $0<\delta<\gamma$. If $h(x)=x$ then any number is a splitting number for $x$.

Definition 4.3. Define $T_{\delta}[x]=\cup\left\{B_{\delta}^{s}(x) \mid s=0, \ldots,(\right.$ ord $\left.x)-1\right\}$ and $S_{\delta}[x]=$ $\left\{B_{\delta}^{s}(x) \mid s=0, \ldots,(\right.$ ord $\left.x)-1\right\}$.

Note that $T_{\delta}\left[h^{s}(x)\right]=T_{\delta}[x]=h^{s}\left(T_{\delta}[x]\right)$ for all $s$.

LEMMA 4.4. If $\delta$ is a splitting number for $x$, then $S_{\delta}[x]$ is an (ord $\left.x\right)$-lift of $\pi\left(T_{\delta}[x]\right)$.

Proof. By the choice of $\delta$, Definition 3.1(i) is satisfied whereas Definition 3.1(ii) is a consequence of

$$
\begin{aligned}
h^{s}\left(B_{\delta}^{i}(x)\right) & =h^{s}\left(B_{\delta}\left(h^{i}(x)\right)\right)=B_{\delta}\left(h^{s+i}(x)\right) \\
& =B_{\delta}\left(h^{s+i \bmod n}(x)\right)=B_{\delta}^{s+i \bmod n}(x) .
\end{aligned}
$$

The observation that if $y \in T_{\delta}[x]$ then $O(y) \subset T_{\delta}[x]$ proves that $\pi^{-1} \pi\left(T_{\delta}[x]\right)=$ $T_{\delta}[x]=\cup S_{\delta}[x]$ or Definition 3.1(iii).

REMARK 4.5. Observe that $T_{\delta}[x]$ is the 1-consolidation of $S_{\delta}[x]$.

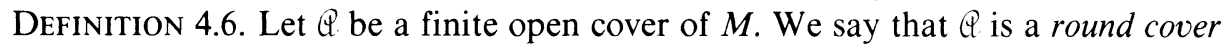
of $M$ iff $\mathbb{Q}=\left\{T_{\gamma_{i}}\left[a_{i}\right] \mid i=1, \ldots, \mu\right\}$ where $a_{i} \in M$ and $\gamma_{i}$ is a splitting number for $a_{i}$.

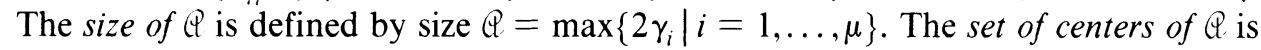
the set $i(\mathbb{Q})=\left\{a_{i} \mid i=1, \ldots, \mu\right\}$.

REMARK 4.7. Observe that any of $a_{i}, h\left(a_{i}\right), \ldots, h^{\left(\text {ord } a_{i}\right)-1}\left(a_{i}\right)$ can serve as the center of $T\left[a_{i}\right]$. Therefore, if $T\left[a_{i}\right] \cap T\left[a_{j}\right] \neq \varnothing$, it is not necessarily true that $B\left(a_{i}\right) \cap B\left(a_{j}\right) \neq \varnothing$. However, once the centers are specified they will remain fixed unless otherwise stated.

Sometimes we will omit the radius $\gamma_{i}$ in order to simplify the notation.

It is easy to get round covers of size less then $\varepsilon$. Simply consider $\left\{T_{\gamma_{x}}[x] \mid x \in M\right\}$ where $\gamma_{x}<\varepsilon$ is a splitting number for $x$ and use the compactness of $M$ to extract a finite subcover.

DEFINITION 4.8. If $Q=\left\{T_{\gamma_{i}}\left[a_{i}\right] \mid i=1, \ldots, \mu\right\}$ is a round cover of $M$ then the Shred of $\mathbb{Q}$ is the finite open cover $\operatorname{Shr} Q=\cup\left\{S_{\gamma_{i}}\left[a_{i}\right] \mid i=1, \ldots, \mu\right\}$.

Informally, $\operatorname{Shr} Q$ is the lift of $\pi(\mathbb{Q})$ formed by the lifts of the elements of $\pi(\mathbb{Q})$, whereas $\mathbb{Q}$ is the "l-consolidation" of $\operatorname{Shr} \mathbb{Q}$.

Notation 4.9. From now on, any time we say $a_{i}$ and $a_{j}$ interlock, we actually mean $S\left[a_{i}\right]$ and $S\left[a_{i}\right]$ interlock. 
Definition 4.10. Let $\mathcal{Q}$ be a round cover of $M$. We say that $\mathcal{Q}$ is a spread cover of $M$ iff whenever $a_{i}, a_{j} \in I(\mathbb{Q})$ are such that $T\left[a_{i}\right] \cap T\left[a_{j}\right] \neq \varnothing$ then $a_{i}$ and $a_{j}$ interlock. We will also say that $a_{i}$ misintersects $a_{j}$ iff $T\left[a_{i}\right] \cap T\left[a_{j}\right] \neq \varnothing$ and $a_{i}$ and $a_{j}$ do not interlock.

Let us now take a look at Figure 4.1 to illustrate the concepts just introduced.

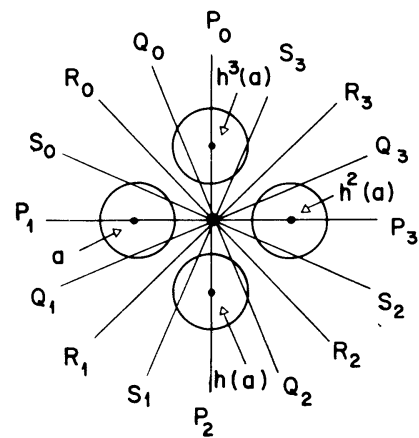

(a) $T[a]$

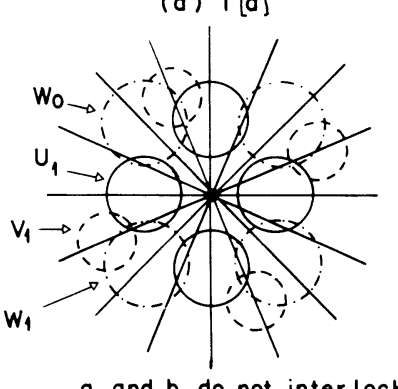

$a$ and $b$ do not inter lock

(d) $a$ and $c$ interlock

$b$ and $c$ interlock

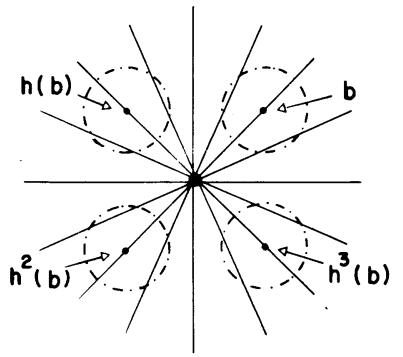

(b) $\mathrm{T}[\mathrm{b}]$

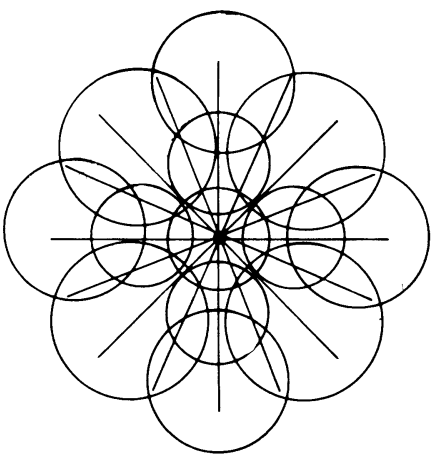

(e) A non-spre od round cover

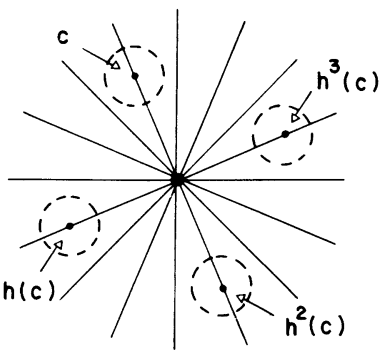

(c) $T[c]$

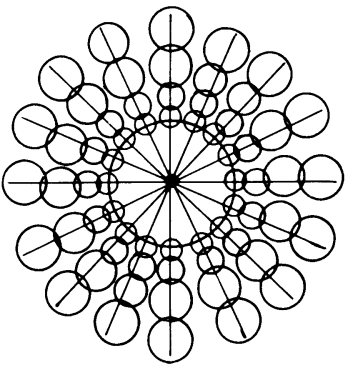

(f) A spread cover

FIGURE 4.1

Let $M$ be the space in the plane consisting of the tree formed by 16 lines joined at one endpoint and with the angle $\pi / 8$ between any two of them. Let $h: M \rightarrow M$ be the homeomorphism defined by a $90^{\circ}$ rotation in the positive direction.

Then, in Figure 4.1(a), the lines $P_{i}, Q_{i}, R_{i}, S_{i}, 0 \leqslant i \leqslant 3$, are mapped by $h$ linearly to the lines $P_{j}, Q_{j}, R_{j}, S_{j}$, respectively, where $j \equiv i+1 \bmod 4$. Observe that under the usual Euclidean metric, $h$ is an isometry.

Let $a \in P_{1}$ with ord $a=4$ and consider the ball $B_{\delta}(a)$ as shown in Figure 4.1(a). Since the interates of this ball are all disjoint, $\delta$ is a splitting number for $a$. Thus $T[a]=\cup\left\{B_{\delta}^{i}(a) \mid i=0, \ldots, 3\right\}$ is a lift of $\pi(T[a])$ of order 4 .

Consider the similar situation for $b \in R_{3}$ and $c \in Q_{0}$ displayed in Figure 4.1(b) and (c). Let us put the three cases together considering $T[a], T[b]$ and $T[c]$ as lifts.

Using Remark 3.2 and Standing Notation 3.15 we can define

$$
\begin{array}{ll}
T[a]=\bigcup\left\{U_{i} \mid i=0, \ldots, 3\right\} & \text { where } U_{i}=B^{i+3 \bmod 4}(a), \\
T[b]=\bigcup\left\{W_{i} \mid i=0, \ldots, 3\right\} & \text { where } W_{i}=B^{i+1 \bmod 4}(b), \\
T[c]=\bigcup\left\{V_{i} \mid i=0, \ldots, 3\right\} & \text { where } V_{i}=B^{i}(c) .
\end{array}
$$


A glance at Definition 3.4 and Figure 4.1(d) is enough to realize that $a$ and $b$ do not interlock, whereas $a$ and $c$, and $b$ and $c$, do. In Figure 4.1(e) and (f) we can compare two round covers which are nonspread and spread, respectively.

We want to prove now the existence of spread covers of arbitrarily small mesh. We will do this by indicating how to obtain a spread cover out of a round cover of convenient mesh.

The method, contained in the proof of the next lemma, consists in replacing (breaking up) all those $T[a]$ 's that produce misintersections by (into) a round refinement $\left\{T\left[\alpha_{k}\right] \mid k=1, \ldots, \mu\right\}$ with $T\left[\alpha_{k}\right]=\cup\left\{Z_{k i}\right\}$, which does not produce any misintersections (see Figure 4.2).

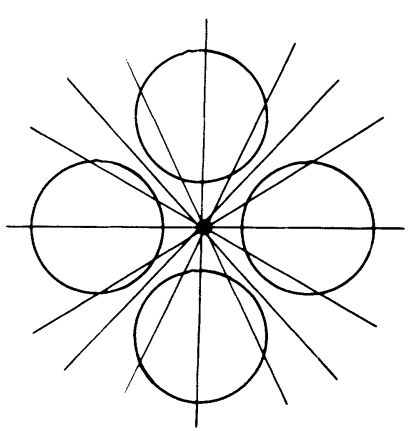

(a) $T[0]$

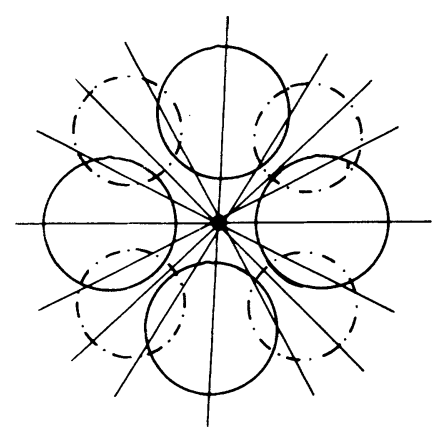

(b) The problem

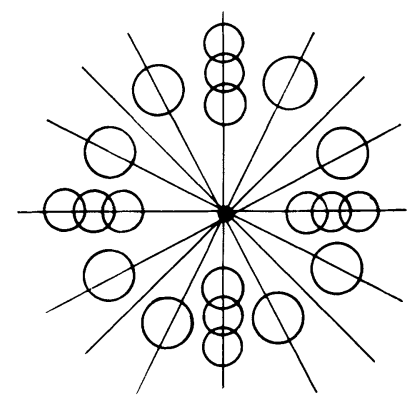

(c) The break-up

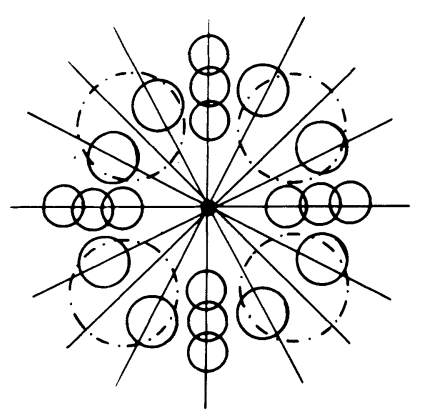

(d) The solution

FIGURE 4.2

LEMMA 4.11. For any round cover $\mathbb{Q}=\left\{T\left[a_{i}\right] \mid i=1, \ldots, \mu\right\}$ of $M$ and any $a_{p} \in I(\mathbb{Q})$ there exist $\alpha_{k} \in M, k=1, \ldots, \mu_{1}$, such that $T\left[a_{p}\right] \subset \cup\left\{T\left[a_{k}\right] \mid k=1, \ldots, \mu_{1}\right\}$ and no $\alpha_{j}$ misintersects any element of $\left\{a_{i} \mid i \neq p\right\} \cup\left\{a_{k} \mid k \neq j\right\}$.

Proof. If ord $a_{i}=1$ for all $i=1, \ldots, \mu$ then there are no misintersections whatsoever ( $Q$ is therefore a spread cover) and the lemma is trivially true. Therefore, let us assume that at least for some $i$, ord $a_{i}>1$.

We will use finite induction on ord $a_{p}$ over the \#-graph $\Gamma_{h}$ (cf. Definition 2.11) partially ordered by " $i<j$ iff $j \mid i$ ”. 
Let $\delta=\min \left\{\operatorname{dist}\left(B^{s}\left(a_{i}\right), B^{t}\left(a_{i}\right)\right) \mid\right.$ ord $a_{i}>1 ; 1 \leqslant s<t<$ ord $\left.a_{i} ; i=1, \ldots, \mu\right\}$. Since ord $a_{i}>1$, for some $i, \delta$ is positive. Cover $\overline{B\left(a_{p}\right)}$ with $\left\{B_{\gamma_{j}}\left(\alpha_{j}\right) \mid j=1, \ldots, \mu_{p}\right\}$ where $\alpha_{j} \in \overline{B\left(a_{p}\right)}$ and $\gamma_{j}$ is a splitting number for $\alpha_{j}$ such that $\gamma_{j}<\delta / 4$. Replace now $T\left[a_{p}\right]$ by $\left\{T_{\gamma_{j}}\left[\alpha_{j}\right] \mid j=1, \ldots, \mu_{p}\right\}$.

Now suppose some $\alpha_{j}$ misintersects some $a_{i}, i \neq p$. This means that $\alpha_{j}$ and $a_{i}$ do not interlock so Theorem 3.24 implies that some $B^{s}\left(\alpha_{j}\right)$ intersects both $B^{s_{1}}\left(a_{i}\right)$ and $B^{s_{2}}\left(a_{i}\right)$ for some $s, s_{1}, s_{2}$. But then (Figure 4.3(a))

$$
d\left(B^{s_{1}}\left(a_{i}\right), B^{s_{2}}\left(a_{i}\right)\right)<\operatorname{diam} B^{s}\left(\alpha_{j}\right)<2 \gamma(j)<\delta / 2<\delta
$$

which is impossible. Then no $\alpha_{j}$ misintersects any $a_{i}, i \neq p$.

But it may be that there are some misintersections among the $\alpha_{j}$ 's. Let us now use the induction argument to get rid of those newly-generated misintersections.

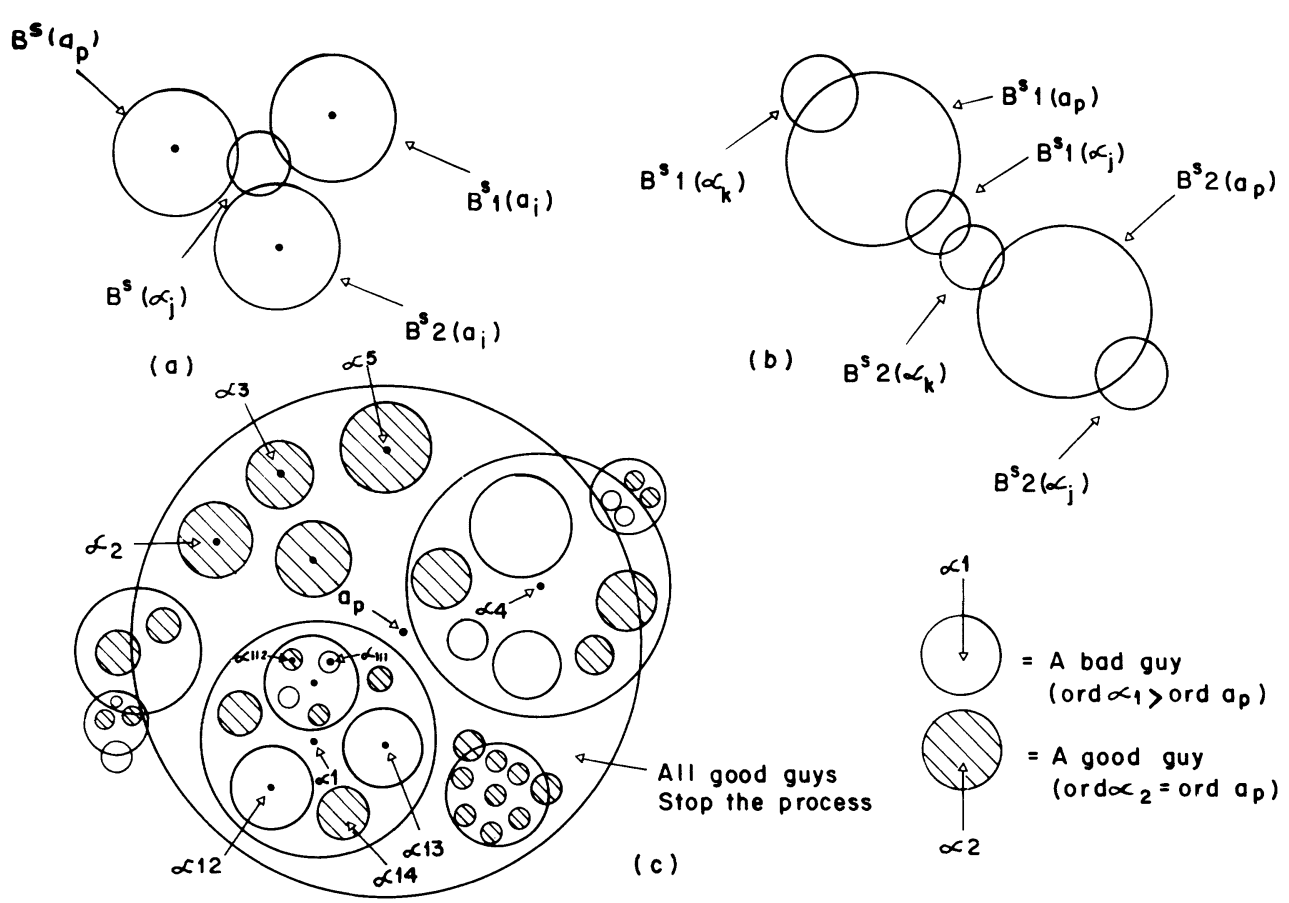

FIGURE 4.3

Initial step. Suppose ord $a_{p}$ is maximal. By Lemma 3.9 ord $a_{p} \mid$ ord $\alpha_{j}$ for all $j$, since $\alpha_{j} \in \overline{B\left(a_{p}\right)}$. But since ord $a_{p}$ is maximal, ord $a_{p}=$ ord $\alpha_{j}$ for all $j$. This means that for all $s$ and all $j, B^{s}\left(\alpha_{j}\right)$ intersects only $B^{s}\left(a_{p}\right)$, otherwise $\operatorname{dist}\left(B^{s}\left(a_{p}\right), B^{s_{1}}\left(a_{p}\right)\right)$ $<\delta$ for some $s_{1}$.

Now suppose that $\alpha_{j}$ misintersects some $\alpha_{k}$. Theorem 3.24 implies that $B^{s_{1}}\left(\alpha_{j}\right)$ intersects $B^{s_{2}}\left(\alpha_{k}\right)$ for some $s_{1} \neq s_{2}$. But then

$$
\begin{aligned}
d\left(B^{s_{1}}\left(a_{p}\right), B^{s_{2}}\left(a_{p}\right)\right) & \leqslant \operatorname{diam} B^{s_{1}}\left(\alpha_{j}\right)+\operatorname{diam} B^{s_{2}}\left(\alpha_{k}\right) \\
& \leqslant 2 \gamma(j)+2 \gamma(k)<\delta / 2+\delta / 2=\delta
\end{aligned}
$$


which is impossible (Figure 4.3(b)). Then no $\alpha_{j}$ misintersects any $\alpha_{k}$ and the argument is completed.

Inductive step (see Figure 4.3(c)). Suppose now that ord $a_{p}$ is not maximal and that the lemma is true for any round cover $\mathscr{B}$ and any $b \in I(\mathscr{T})$ such that ord $a_{p} \mid$ ord $b$ and ord $a_{p} \neq$ ord $b$.

Since $\alpha_{j} \in \overline{B\left(a_{p}\right)}$, Lemma 3.9 tells us that ord $a_{p} \mid$ ord $\alpha_{j}$. Suppose first that ord $\alpha_{j}=$ ord $a_{p}$ and that $\alpha_{j}$ misintersects some $\alpha_{k}$. Again, Theorem 3.24 implies that for some $s, s_{1}, s_{2}, B^{s}\left(\alpha_{k}\right)$ meets both $B^{s_{1}}\left(\alpha_{j}\right)$ and $B^{s_{2}}\left(\alpha_{j}\right)$ making the distance between $B^{s_{1}}\left(a_{p}\right)$ and $B^{s_{2}}\left(a_{p}\right)$ less than $\delta$, a contradiction. Then we do not have to worry about $a_{j}$ 's with ord $a_{j}=$ ord $a_{p}$, so if ord $a_{j}=$ ord $a_{p}$ for all $j$ then we are done.

The only problems now are the $\alpha_{j}$ 's such that ord $a_{p} \mid$ ord $\alpha_{j}$ and ord $a_{p} \neq$ ord $\alpha_{j}$. Let us rename them as $\alpha_{i}, i=1, \ldots, l$. Apply now the induction hypothesis to the round cover $\left\{T\left[\alpha_{j}\right] \mid j=1, \ldots, l\right\} \cup\left\{T\left[a_{i}\right] \mid i \neq p\right\}$ and $\alpha_{1}$ to obtain a new round cover $\left\{T\left[\alpha_{1 k}\right]\right\} \cup\left\{T\left[\alpha_{j}\right] \mid j \neq 1\right\} \cup\left\{T\left[a_{i}\right] \mid i \neq p\right\}$ where no $\alpha_{1 k}$ misintersects any other element. Do the same now for this cover and $\alpha_{2}$ to obtain another round cover $\left\{T\left[\alpha_{1 k}\right]\right\} \cup\left\{T\left[\alpha_{2 k}\right]\right\} \cup\left\{T\left[\alpha_{j}\right] \mid j \neq 1,2\right\} \cup\left\{T\left[a_{i}\right] \mid i \neq p\right\}$ where no $\alpha_{1 k}$ and no $\alpha_{2 k}$ misintersect any other element.

Proceed this way with all the $\alpha_{i}, i=1, \ldots, l$. The final cover so obtained satisfies the conditions of the statement of the lemma.

REMARK 4.12. Observe that a spread cover is one in which no element misintersects any other element. With this in mind we proceed to prove the main result of this section.

THEOREM 4.13. Given $\varepsilon>0$, there exists a spread cover $Q$ such that size $Q<\varepsilon$.

Proof. Start with any round $\operatorname{cover} \Theta=\left\{T\left[a_{i}\right] \mid i=1, \ldots, \mu\right\}$ of size less than $\varepsilon$.

Consider $a_{1} \in I(\mathbb{Q})$. Use the previous lemma to break up $T\left[a_{1}\right]$ into $\left\{T_{\gamma_{1 k}}\left[\alpha_{1 k}\right] \mid k\right.$ $\left.=1, \ldots, \mu_{1}\right\}$ where $\gamma_{1 k}<\varepsilon / 2$. We obtain a new round cover $\left\{T\left[\alpha_{1 k}\right] \mid k=1, \ldots, \mu_{1}\right\}$ $\cup\left\{T\left[a_{i}\right] \mid i=2, \ldots, \mu\right\}$ in which no $\alpha_{1 k}$ misintersects any other element.

Now look at $\alpha_{2}$ and use the last lemma again to obtain another round cover

$$
\left\{T\left[\alpha_{1 k}\right] \mid k=1, \ldots, \mu_{1}\right\} \cup\left\{T\left[\alpha_{2 k}\right] \mid k=1, \ldots, \mu_{2}\right\} \cup\left\{T\left[a_{i}\right] \mid i=3, \ldots, \mu\right\}
$$

of size less than $\varepsilon$ and such that no element misintersects any other element.

Continuing in this fashion we end up with a round cover $\mathscr{Q}=\left\{T\left[\alpha_{i k}\right] \mid i=1, \ldots, \mu\right.$; $\left.k=1, \ldots, \mu_{i}\right\}$ of size less than $\varepsilon$ and such that no element misintersects any other element.

$Q$ is therefore a spread cover of size less than $\varepsilon$.

REMARK 4.14. Suppose that $U \subset M_{h}$ is such that $U<\pi(Q)$, where $Q$ is a spread cover of $M$. Consider $H=\left\{a_{i} \in I(\mathbb{Q}) \mid U \subset \pi\left(T\left[a_{i}\right]\right)\right\}$. Observe that $a_{i}, a_{j} \in H$ implies that $T\left[a_{i}\right] \cap T\left[a_{j}\right] \neq \varnothing$ and since $\mathscr{Q}$ is spread, $a_{i}$ and $a_{j}$ interlock. Therefore the set $\left\{\right.$ ord $\left.a_{i} \mid a_{i} \in H\right\}$ is pairwise divisible. Furthermore, by Corollary 3.23, $\left\{L\left(U, S\left[a_{i}\right]\right) \mid a_{i} \in H\right\}$ is such that out of every pair of lifts, one is a consolidation of the other. That is, it is a totally ordered set under refinement, using Lemma 3.6. Therefore there is a maximal element. 
This allows us to have, without ambiguity, the following

DEFINITION 4.15. Let $\Theta$ be a spread cover of $M$ and $U<\pi(\Theta)$ where $U$ is an open set. Define the lift of $U$ induced by $Q, L(U, \mathbb{Q})$, by $L(U, \mathbb{Q})=L\left(U, S\left[a_{i}\right]\right)$ where ord $a_{i}=\max \left\{\operatorname{ord} a_{j} \mid U \subset \pi\left(T\left[a_{j}\right]\right)\right\}$. If $m \mid$ ord $a_{i}, L_{m}(U, \mathbb{Q})$ denotes the $m$-consolidation of $L(U, \mathbb{Q})$. If $C=\left\{C_{i}\right\}$ is a finite open cover of $M_{h}$ such that $C<\pi(\mathbb{Q})$, then the lift of $C$ induced by $\mathbb{Q}, L(C, \mathbb{Q})$, is defined to be the cover $L(C, \mathbb{Q})=$ $\cup\left\{L\left(C_{i},(\varphi) \mid C_{i} \in C\right\}\right.$.

REMARK 4.16. It follows from this definition that if $U \subset \pi(T[a]), a \in I(\Theta)$, then $L(U, S[a])=L_{\text {ord } a}(U, \mathbb{Q})$ and that, by Lemma 3.7, if $p \mid$ ord $a$ then, $L_{p}(U, S[a])=$ $L_{p}(U, \mathbb{Q})$.

THEOREM 4.17. Let $\Theta$ be a spread cover of size less than $\varepsilon$ and $C<\pi(\Theta)$ a finite open cover of $M_{h}$. Then $L(C, \mathbb{Q})$ is a finite open cover of $M$, invariant under $h$, of mesh less than $\varepsilon$. Furthermore, $\operatorname{dim} L(C, \mathbb{Q})=\operatorname{dim} C$.

Proof. Let $U \in L(C, \mathbb{Q})$. Then $U<S[a]$ for some $a \in I(\mathbb{Q})$ so $U \subset B_{\gamma}^{s}(a)$ for some $s$. Therefore $\operatorname{diam} U<\operatorname{diam} B_{\gamma}^{s}(a)<2 \gamma \leqslant \operatorname{size} Q<\varepsilon$ and so mesh $L(C, \mathbb{Q})<\varepsilon$.

Now let $U, V \in C$, such that $U \cap V \neq \varnothing$. Suppose that $a, b \in I(\mathbb{Q})$ are centers of maximal orders such that $U \subset \pi(T[a])$ and $V \subset \pi(T[b])$, respectively. Then $T[a] \cap T[b] \neq \varnothing$ and since $\Theta$ is spread, $a$ and $b$ interlock. Then $L(U, \mathbb{Q})$ and $L(V, \mathbb{Q})$ or, equivalently, $L(U, S[a])$ and $L(V, S[b])$ interlock, following Lemma 3.18 .

All we have to do now is to apply Lemma 3.25 to conclude the proof.

THEOREM 4.18. Let $Q$ be a spread cover of $M$. If $C<\pi(\Theta)$ is a tree-cover of $M_{h}$, then $\Re(L(C, \mathbb{Q}))$ is a bellows. Furthermore $\bar{h}: \mathcal{X}(L(C, \mathbb{Q})) \rightarrow \mathscr{N}(L(C, \mathbb{Q}))$, the map induced by $h$, is an isomorphism which is also an isometry.

Proof. Use the previous lemma in conjunction with Lemma 3.26.

REMARK 4.19. We can use again Example 3.27 to show the necessity of $C$ being a tree-cover, by noticing that the covers used in that example were a spread cover and its projection.

Corollary 4.20. Let $h: M \rightarrow M$ be a periodic homeomorphism such that $M_{h}$ is tree-like. Then, for every $\varepsilon>0$, there exists a finite open cover $C$ of $M$ of mesh less than $\varepsilon$, invariant under $h$ and such that $\Re(C)$ is a 1-dimensional bellows.

Proof. First, get a spread cover $Q$ of size less than $\varepsilon$. Then use the Lebesgue number of $\pi(\mathbb{Q})$ and the fact that $M_{h}$ is tree-like to find a tree-cover $C$ of $M_{h}$ such that $C<\pi(\Theta)$. Finally, apply Theorems 4.17 and 4.18.

Now we want to generalize Theorems 4.17 and 4.18 in the following way.

Definition 4.21. Let $Q=\left\{T\left[a_{i}\right] \mid i=1, \ldots, \mu\right\}$ be a spread cover of $M$ and $C=\left\{C_{i} \mid i=1, \ldots, \lambda\right\}$ a tree-cover of $M_{h}$ such that $C<\pi(\mathbb{Q})$. Let $\sigma: C \rightarrow N^{+}$be a divisible \#-graph such that $\sigma\left(C_{i}\right) \mid$ ord $L\left(C_{i}, \mathbb{Q}\right)$ for all $C_{i} \in C$. Define the $\sigma$-lift of $C$ induced by $\mathbb{Q}, L_{\sigma}(C, \mathbb{Q})$, by $L_{\sigma}(C, \mathbb{Q})=\cup\left\{L_{\sigma\left(C_{i}\right)}\left(C_{i}, \mathbb{Q}\right) \mid C_{i} \in C\right\}$.

THEOREM 4.22. Let $Q$ be a spread cover of $M, C<\pi(\mathbb{Q})$ a finite open cover of $M_{h}$ and $(C, \sigma)$ a divisible \#-graph such that $\sigma\left(C_{i}\right) \mid$ ord $L\left(C_{i}, \mathbb{Q}\right)$. Then $L_{\sigma}(C, \mathbb{Q})$ is a finite open cover of $M$ invariant under $h$ and $\operatorname{dim} L_{\sigma}(C, \mathbb{Q})=\operatorname{dim} C$. 
Proof. Since $(C, \sigma)$ is divisible, if $C_{i}, C_{j} \in C$ are such that $C_{i} \cap C_{j} \neq \varnothing$ then $\{\sigma(i), \sigma(j)\}$ is divisible. Then, by Corollary $3.21, L_{\sigma\left(C_{i}\right)}\left(C_{i}, \mathcal{\leftrightarrow}\right)$ and $L_{\sigma\left(C_{1}\right)}\left(C_{j}, \mathbb{Q}\right)$ interlock. Now apply Lemma 3.25.

REMARK 4.23. Notice that in this case size $\Theta<\varepsilon$ does not necessarily imply mesh $L_{\sigma}(C, \mathbb{Q})<\varepsilon$ since some elements of $L_{\sigma}(C, \mathbb{Q})$ may be unions of elements of Shr $\Theta$ too far apart (recall that if $\sigma\left(C_{i}\right) \neq$ ord $L\left(C_{i}, \Theta\right)$, then we are performing consolidations).

The next theorem is the analogue of Theorem 4.18 and follows from Lemmas 3.21 and 3.26 .

THEOREM 4.24. Let $\uparrow$ be a spread cover of $M, C<\pi(\uparrow)$ a tree-cover of $M_{h}$ and $(C, \sigma)$ a divisible \#-graph such that $\sigma\left(C_{i}\right) \mid \operatorname{ord} L\left(C_{i}, \mathcal{Q}\right)$. Then $\mathcal{O}\left(L_{\sigma}(C, \mathbb{Q})\right)$ is the $(C, \sigma)$-bellows and $\bar{h}$, the map induced by $h$ on $\Re\left(L_{\sigma}(C, \mathbb{Q})\right)$, is an isomorphism which is also an isometry.

Observe that Theorems 4.17 and 4.18 are particular cases of the two previous theorems, namely for the case when $\sigma\left(C_{i}\right)=\operatorname{ord} L\left(C_{i}, \mathbb{Q}\right)$. We can then refer in general to $L_{\sigma}(C, Q)$.

LEMMA 4.25. Let $\Theta$ be a spread cover of $M, C<\pi(\Theta)$ a tree-cover of $M_{h},(C, \sigma) a$ divisible \#-graph such that $\sigma\left(C_{i}\right) \mid$ ord $L\left(C_{i}, \mathbb{Q}\right)$ and $\varepsilon>0$. Then $N=\Re\left(L_{\sigma}(C, \mathbb{Q})\right)$ has a simplical subdivision $N^{\prime}$ of diameter less than $\varepsilon$ that is invariant under $\bar{h}$. That is, if $p$ is a vertex of $N^{\prime}$, then $\bar{h}(p)$ also is.

Proof. Recall that the vertices of $N$ are of the form $U_{E i}$, where $E \in C$ and $0 \leqslant i<\sigma(E)$.

Suppose that $\left(U_{E i}, U_{D j}\right)$ is an edge of $N$ so, say, $\sigma(E) \mid \sigma(D)$ and $i \equiv j \bmod \sigma(E)$. Then $h^{j}\left(U_{E 0}\right)=U_{E(j \bmod \Sigma(E))}=U_{E i}$ and $h^{j}\left(U_{D 0}\right)=U_{D j}$. Then $\bar{h}^{j}\left(\left(U_{E 0}, U_{D 0}\right)\right)=$ $\left(E_{E i}, U_{D j}\right)$, since $\bar{h}$ is a linear extension of $h$.

Now consider the subgraphs $\bar{N}$ of $N$ generated by all those vertices of $N$ labeled with " 0 ". By the previous discussion, $\bar{N} \cup h(\bar{N}) \cup \cdots \cup h^{n}(\bar{N})=N$. Find a subdivision $\bar{N}^{\prime}$ of $\bar{N}$ of diameter less than $\varepsilon$. Since, by Theorem $4.24 \bar{h}$ is an isometry, $N^{\prime}=\bar{N}^{\prime} \cup h\left(\bar{N}^{\prime}\right) \cup \cdots \cup h^{n}\left(\bar{N}^{\prime}\right)$ is a simplical subdivision of $N$ of diameter less than $\varepsilon$. Clearly, $N^{\prime}$ is invariant under $\bar{h}$ (see Figure 4.4).

5. Inducing periodic homeomorphisms. In this section we investigate the conditions under which a periodic homeomorphism of a continuum onto itself can be induced as the limit of an inverse sequence of homeomorphisms.

We also investigate the kinds of spaces that the resulting inverse sequences are composed of.

Throughout this section $M$ denotes a metric continuum, and if $h$ is a periodic homeomorphism of $M$ onto itself, $M_{h}$ denotes the orbit space of $M$ under $h$ and $\pi$ : $M \rightarrow M_{h}$ the projection map.

Our main results for this section are Theorems 5.3, 5.13 and 5.15, and Example 5.17 .

We start the sections with a basic definition. 

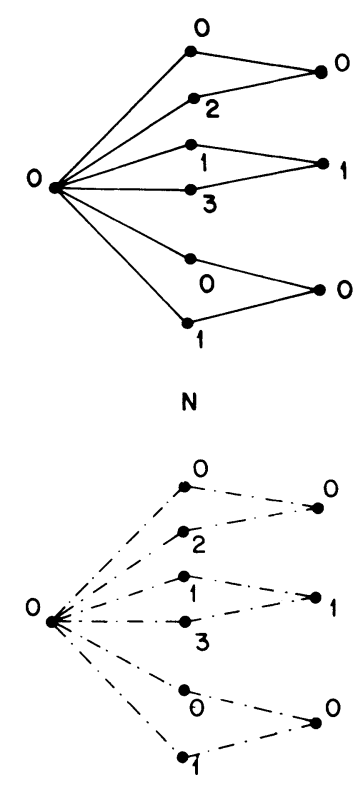

$N^{\prime}=N^{\prime} \cup h\left(\bar{N}^{\prime}\right) \cup h^{2}\left(\bar{N}^{\prime}\right) \cup h^{3}\left(\bar{N}^{\prime}\right)$

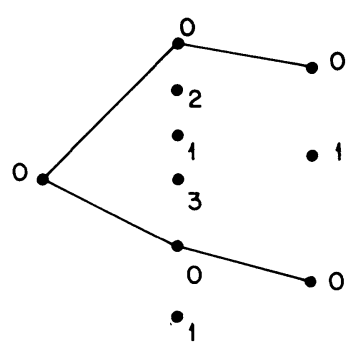

$\bar{N}$

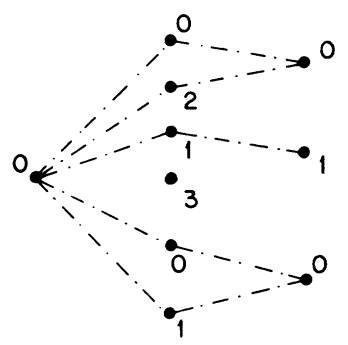

$\bar{N}^{\prime} \cup h\left(\bar{N}^{\prime}\right) \cup h^{2}\left(\bar{N}^{\prime}\right)$

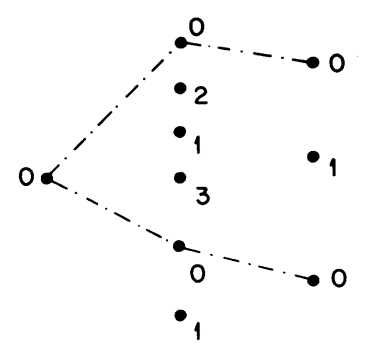

$\bar{N}^{\prime}$

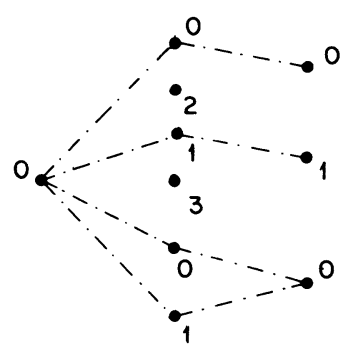

$\bar{N}^{\prime} \cup h\left(\bar{N}^{\prime}\right)$

FIGURE 4.4

Definition 5.1. A homeomorphism $h: M \rightarrow M$ is inducible iff there exists a commutative diagram

$$
\begin{array}{lccccccccc}
P_{1} & \stackrel{\alpha_{1}}{\leftarrow} & P_{2} & \stackrel{\alpha_{2}}{\leftarrow} & P_{3} & \stackrel{\alpha_{3}}{\leftarrow} & \cdots & P & \stackrel{\alpha}{\rightarrow} & M \\
f_{1} \downarrow & & f_{2} \downarrow & & f_{3} \downarrow & & & f \downarrow & & \downarrow h \\
Q_{1} & \stackrel{\beta_{1}}{\leftarrow} & Q_{2} & \stackrel{\beta_{2}}{\leftarrow} & Q_{3} & \stackrel{\beta_{3}}{\leftarrow} & \ldots & Q & \stackrel{\beta}{\rightarrow} & M
\end{array}
$$

where $P=\lim \left\{P_{i}, \alpha_{i}\right\}, Q=\lim \left\{Q_{i}, \beta_{i}\right\}, P_{i}$ and $Q_{i}$ are finite simplicial complexes, $\alpha$ and $\beta$ are homeomorphisms, $\overleftarrow{f}_{i}$ are simplical homeomorphisms, and $f$ is the homeomorphism induced by $\left\{f_{i}\right\}$. If in addition $\alpha=\beta$ (and necessarily $P_{i}=Q_{i}$ and $P=Q) h$ is said to be strongly inducible.

$\mathrm{Kaul}[\mathbf{K}]$ proved that any homeomorphism of compact metric spaces is inducible. This was accomplished by constructing a commutative diagram

$$
\begin{array}{cccccccc}
\Re\left(\mathscr{Q}_{1}\right) & \stackrel{\pi_{1}}{\leftarrow} & \Re\left(\mathscr{Q}_{2}\right) & \stackrel{\pi_{2}}{\leftarrow} & \cdots & P & \stackrel{\alpha}{\rightarrow} & M \\
\downarrow f_{1} & & \downarrow f_{2} & & & f \downarrow & & \downarrow h \\
\Re\left(\mathscr{V}_{1}\right) & \stackrel{\phi_{1}}{\leftarrow} & \Re\left(\mathscr{V}_{2}\right) & \stackrel{\phi_{2}}{\leftarrow} & \ldots & Q & \stackrel{\beta}{\rightarrow} & M
\end{array}
$$

where $\mathscr{U}_{i}$ are finite open covers of $M$ of mesh less than $1 / 2^{i}, \mathscr{V}_{i}=h\left(\mathcal{U}_{i}\right), \Re(\mathscr{U})$ denotes the nerve of $\mathscr{Q}$ and $f_{i}$ are the simplicial maps induced by $h$.

Definition 5.2. A homeomorphism $h: M \rightarrow M$ is $B$-inducible iff:

(i) $h$ is strongly inducible.

(ii) For all $i, P_{i}$ in Definition 5.1 is a bellows. 
Using the material of $\S \S 3$ and 4 which is an elaboration of the techniques developed by Fugate and McLean [FM] and referring to the work by Kaul [K], we proceed to prove the following

THEOREM 5.3. Let $h: M \rightarrow M$ be a periodic homeomorphism of a metric continuum $M$ such that $M_{h}$ is tree-like. Then $h$ is B-inducible.

Proof. We will follow very closely the proof of Theorem 4 in $[\mathbf{K}]$. For definitions of the concepts used in this proof refer to [K].

Use the fact that $M_{h}$ is tree-like and Corollary 4.20 to find an invariant finite open cover $\mathcal{Q}_{1}$ of $M$ of mesh less than $\frac{1}{2}$ such that $\Re\left(\mathcal{U}_{1}\right)$ is a bellows.

Let $f_{1}: \Re\left(\mathscr{U}_{1}\right) \rightarrow \Re\left(\mathscr{U}_{1}\right)$ be the homeomorphisms induced by $h$. Since $h$ is an isometry, we can construct a barycentric map [K, p. 297], $\alpha_{1}: M \rightarrow \mathfrak{\mathcal { U }}\left(\mathscr{Q}_{1}\right)$ such that $f_{1} \alpha_{1}=\alpha_{1} h$.

Use Lemma 4.25 to find a simplicial subdivison $\Re\left(\mathscr{U}_{1}\right)^{\prime}$ of $\Re\left(\mathscr{U}_{1}\right)$ of mesh less than $\frac{1}{2}$, invariant under $f_{1}$. Then

$$
h\left(\alpha_{1}^{-1}(\operatorname{st}(p))\right)=\alpha^{-1}\left(\operatorname{st}\left(f_{1}(p)\right)\right)
$$

where $p$ is any vertex of $\mathscr{\varkappa}\left(\mathscr{U}_{1}\right)^{\prime}$ and the open stars are taken with respect to $\Re\left(\mathscr{U}_{1}\right)^{\prime}$.

Let $\mathscr{U}_{1.5}$ be the auxiliary cover [K, (1.1)], of $M$ with respect to $\alpha_{1}$ and $\Re\left(\mathscr{U}_{1}\right)^{\prime}$. $\mathcal{U}_{1.5}$ is invariant under $h$ so $f_{1.5}: \mathcal{X}\left(\mathcal{U}_{1.5}\right) \rightarrow \mathcal{X}\left(\mathcal{Q}_{1.5}\right)$, the map induced by $h$, is an isomorphism. Furthermore, the diagram

$$
\begin{array}{ccc}
\Re\left(\mathcal{U}_{1}\right)^{\prime} & \stackrel{i_{1}}{\leftarrow} & \Re\left(\mathcal{U}_{1.5}\right) \\
\downarrow f_{1} & & \downarrow f_{1.5} \\
\Re\left(\mathcal{U}_{1}\right)^{\prime} & \stackrel{i_{1}}{\leftarrow} & \Re\left(\mho_{1.5}\right)
\end{array}
$$

commutes, where $i_{1}$ is a simplicial isomorphism [ $\mathbf{K}$, Lemma 2.1].

Again use Corollary 4.20 to find an invariant finite open cover $\mathscr{Q}_{2}$ of $M$ of mesh less than $1 / 2^{2}$ and such that $\overline{\partial_{2}}<\mathcal{U}_{1.5}$ and $\mathcal{T}\left(\mathcal{U}_{2}\right)$ is a bellows. Furthermore, if $f_{2}$ : $\mathscr{R}\left(\mathscr{U}_{2}\right) \rightarrow \mathscr{N}\left(\mathscr{U}_{2}\right)$ is the homeomorphism induced by $h$, then, since $\mathfrak{X}\left(\mathscr{U}_{2}\right)$ is a bellows, we can construct a projection map $[\mathbf{K},(1.3)] \pi_{1}^{\prime}: \mathfrak{\Re}\left(\mathscr{U}_{2}\right) \rightarrow \mathfrak{N}\left(\mathscr{U}_{1.5}\right)$ in such a way that the diagram

$$
\begin{array}{ccc}
\Re\left(\mathcal{U}_{1.5}\right) & \stackrel{\pi_{1}^{\prime}}{\leftarrow} & \Re\left(\mathcal{U}_{2}\right) \\
\downarrow f_{1.5} & & \downarrow f_{2} \\
\Re\left(\cup_{1.5}\right) & \stackrel{\pi_{1}^{\prime}}{\leftarrow} & \Re\left(\mathcal{U}_{2}\right)
\end{array}
$$

commutes. 
Define $\pi_{1}=i_{1} \circ \pi_{1}^{\prime}$ and put together the two previous diagrams to obtain the following commutative diagram:

$$
\begin{array}{ccc}
\Re\left(\mho_{1}\right)^{\prime} & \stackrel{\pi_{1}}{\leftarrow} & \Re\left(\vartheta_{2}\right) \\
\downarrow f_{1} & & \downarrow f_{2} \\
\Re\left(\mho_{1}\right)^{\prime} & \stackrel{\pi_{1}}{\leftarrow} & \Re\left(\vartheta_{2}\right)
\end{array}
$$

Since $\Re\left(\mathscr{U}_{1}\right)^{\prime}$, as a space, is the same as $\mathscr{N}\left(\mathscr{U}_{1}\right)$ we actually obtained a commutative diagram:

$$
\begin{array}{ccc}
\Re\left(\mho_{1}\right) & \stackrel{\pi_{1}}{\leftarrow} & \Re\left(\mho_{2}\right) \\
\downarrow f_{1} & & \downarrow f_{2} \\
\Re\left(\mho_{1}\right) & \stackrel{\pi_{1}}{\leftarrow} & \Re\left(\vartheta_{2}\right)
\end{array}
$$

Continue in this fashion to obtain:

$$
\begin{aligned}
& \Re\left(\mathscr{U}_{1}\right) \stackrel{\pi_{1}}{\leftarrow} \Re\left(\mathscr{U}_{2}\right) \stackrel{\pi_{2}}{\leftarrow} \Re\left(\mathscr{U}_{3}\right) \leftarrow \ldots P \stackrel{\alpha}{\rightarrow} M \\
& \downarrow f_{1} \quad f_{2} \downarrow \quad f_{3} \downarrow \quad \downarrow f \quad \downarrow h \\
& \Re\left(\mathscr{Q}_{1}\right) \stackrel{\pi_{1}}{\leftarrow} \Re\left(\mathscr{U}_{2}\right) \stackrel{\pi_{2}}{\leftarrow} \Re\left(\mathscr{Q}_{3}\right) \leftarrow \ldots P \stackrel{\alpha}{\rightarrow} \quad M
\end{aligned}
$$

Since for all $i, \Re\left(\mathcal{Q}_{i}\right)$ is a bellows, $h$ is $B$-inducible.

COROLlaRY 5.4. A periodic homeomorphism $h$ is B-inducible iff for every $\varepsilon>0$ there exists an invariant bellow-cover of $M$ of mesh less than $\varepsilon$.

Proof. This follows from the proof of Theorem 5.3.

There are several ways of improving the result in Theorem 5.3 as indicated in the following

Definition 5.5. A $B$-inducible homeomorphism $h$ is $E$-inducible iff for all $i, j, P_{i}$ and $P_{j}$ of Definition 5.1 are homeomorphic. It is $T$-inducible iff each $P_{i}$ is a tree. Finally, it is $E T$-inducible iff it is both $E$ - and $T$-inducible.

Naturally, we have the following

QUESTION 5.6. What conditions on $M, M_{h}$ and $h$ are necessary and sufficient in order for a periodic homeomorphism $h$ to be $E$-, $T$-, or $E T$-inducible?

From Corollary 5.4 we see that the existence of invariant bellows-covers of arbitrarily small mesh with homeomorphic nerves implies $E$-inducibility. Similarly, if these covers are tree-covers we obtain $T$-inducibility. Of course, in order to obtain $T$-inducibility $M$ must be tree-like.

A stronger way of inducing periodic homeomorphisms can be described as follows. Recall the definition of the \#-graph associated with a periodic homeomorphism (Definition 2.11).

Definition 5.7. A periodic homeomorphism $h$ with \#-graph $\Gamma$ is called ( $\Gamma-)$ perfect iff it is $E$-inducible and all the factor spaces in the inverse sequence are either $\Gamma$-bellows or $(1+\Gamma)$-bellows. 


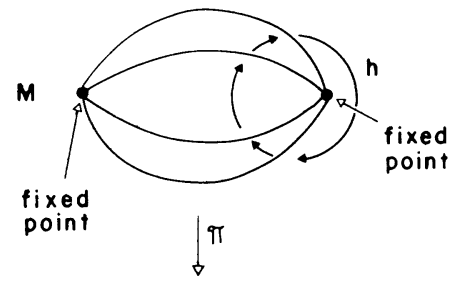

$m_{n}$

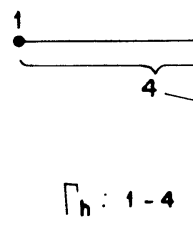

(a)

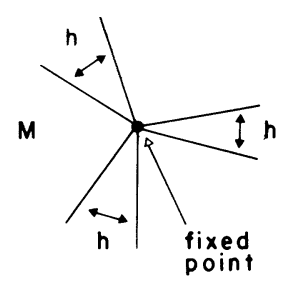

$\pi$

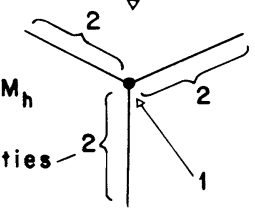

$\Gamma_{h}: 1-2$

(b)
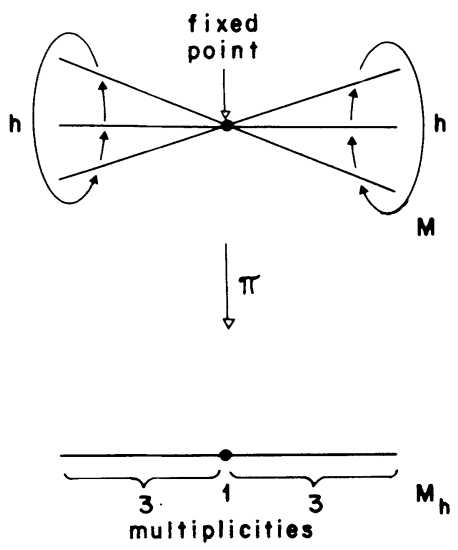

$\Gamma_{n}: 1-3$

(c)

Non-perfect Periodic Homeomorphisms

FIGURE 5.1

It is easy to find examples of perfect homeomorphisms for some admissible $\Gamma$ (see Definition 2.11) as can be seen from Remark 2.21.

In Figure 5.1 we have examples of periodic homeomorphisms which are not perfect. All these homeomorphisms share the property of having orbit spaces "not related" to their \#-graph. We proceed to introduce this notion more formally.

Definition 5.8. Let $C=\left\{C_{i} \mid i=1, \ldots, \mu\right\}$ be an open finite cover of $M_{h}$. The natural \#-graph of $C$ is defined as $\left(C, \sigma_{c}\right)$ where $\sigma_{c}\left(C_{i}\right)=\min \left\{\right.$ mult $\left.x \mid x \in C_{i}\right\}$. A number $\varepsilon>0$ is called critical for $h$ iff for any finite open cover $C$ of mesh less than $\varepsilon$, the set $\left\{\sigma_{c}\left(C_{i}\right) \mid C_{i} \in C\right\}$ equals $\Gamma_{h}$.

Definition 5.9. Let $h$ be a divisible homeomorphism with \#-graph $\Gamma_{h}$. We say that $M_{h}$ is perfect iff for every $\varepsilon>0$ there exist a cover $C$ of mesh less than $\varepsilon$ and such that $\left(C, \sigma_{c}\right)=1+\cdots+\Gamma_{n}$ (see Definition 2.17).

So to speak, $M_{h}$ is perfect iff it is the "inverse limit of $\Gamma_{h}$ 's". Observe how the orbit spaces of the examples in Figure 5.1 are not perfect.

We want to describe a situation that forces orbit spaces to be perfect.

Standing Assumptions 5.10. In what follows, we will be assuming that $M$ is tree-like, $M_{h}$ is chainable and that $h$ is a divisible periodic homeomorphism.

Recall from $\S 1$ that if $h$ is divisible, then $M=\cup\left\{F_{i} \mid i \in \Gamma_{h}\right\}, F_{1}=F_{n_{1}} \subset F_{n_{2}} \subset$ $\cdots \subset M$ where $\Gamma_{h}$ is the \#-graph of $h$, i.e., $\Gamma_{h}=\{m \in Z \mid m=$ ord $x$ for some $x \in M\}=\left\{1=n_{1}<n_{2}<\cdots<n_{p}\right\}$ and for $i \in \Gamma_{h}, F_{i}=\{x \mid$ ord $x$ divides $i\}$ is a continuum. Then $M_{h}=\bigcup\left\{\pi\left(F_{i}\right) \mid i \in \Gamma_{h}\right\}$ and $\pi\left(F_{1}\right) \subset\left(F_{n_{2}}\right) \subset \cdots \subset M_{h}$.

LEMMA 5.11. A critical number for $h$ always exists.

Proof. Let us find one. For each $1 \leqslant i \leqslant p$ let $x_{i} \in M_{h}$ be such that mult $x_{i}=n_{i}$, $n_{i} \in \Gamma_{h}$. Then $x_{i} \notin F_{n_{i-1}}$. Let $\varepsilon<\frac{1}{2} \min \left\{\operatorname{dist}\left(x_{i}, F_{n_{i-1}}\right) \mid i=2, \ldots, p\right\}$. We claim that $\varepsilon$ 
is a critical number for $h$. For if $C$ is a chain of mesh less than $\varepsilon$, then, for every $1 \leqslant i \leqslant p$, there exists a $C_{s_{i}} \in C$ such that $x_{i} \in C_{s_{i}}$ and $C_{s_{i}} \cap F_{n_{i-1}}=\varnothing$. Thus $\sigma_{c}\left(C_{s_{i}}\right)=n_{i}$ so $\sigma_{c}(C)=\Gamma_{h}$.

LEMMA 5.12. Let $\varepsilon>0$ be a critical number for $h$ and $D=\left\{D_{i} \mid i=1, \ldots, \lambda\right\}$ be $a$ chain-cover of $M_{h}$ of mesh less than $\varepsilon$ such that $D_{1} \cap \pi\left(F_{1}\right) \neq \varnothing$. Then there exist integers $0=q_{0}<q_{1}<\cdots<q_{p}=\lambda$ such that $\sigma_{c}\left(D_{i}\right)=n_{j}$ iff $q_{j-1}<i \leqslant q_{j}$.

Proof. If we denote by $E_{i}$ the subcover of $D$ that intersects $\pi\left(F_{n_{i}}\right)$ then, since $\pi\left(F_{n_{i}}\right)$ is connected, $E_{i}$ is a subchain of $D$. Because $D_{1} \in E_{1}$ and $\pi\left(F_{1}\right) \subset \pi\left(F_{n_{2}}\right) \subset$ $\cdots \subset \pi\left(F_{n_{p}}\right)=M_{h}$, we must have that $E_{1} \subset E_{2} \subset \cdots \subset E_{p}$. Set $q_{i}$ equal to the index of the last link on $E_{i}$. The lemma follows.

THEOREM 5.13. There exists an $\varepsilon>0$ such that if $C$ and $D$ are any two chain-covers of $M_{h}$ of mesh less than $\varepsilon$ with natural \#-graphs $\sigma_{C}$ and $\sigma_{D}$, respectively, and such that $C_{1}$ and $D_{1}$ intersect $\pi\left(F_{1}\right)$, then the $\left(C, \sigma_{c}\right)$-bellows and the $\left(D, \sigma_{D}\right)$-bellows are homeomorphic. Furthermore, they are homeomorphic to the $\Gamma_{h}$-bellows, if $F_{1}$ is a singleton, and to the $\left(1+\Gamma_{h}\right)$-bellows, otherwise.

Proof. Let $\varepsilon>0$ be a critical number for $h$ such that $\varepsilon<\frac{1}{2} \operatorname{diam} \pi\left(F_{1}\right)$ if $\operatorname{diam} \pi\left(F_{1}\right) \neq 0$. By this choice of $\varepsilon$, Lemma 5.12 is telling us that if $C$ is a chain cover of $M_{h}$ of mesh less than $\varepsilon$ starting at $\pi\left(F_{1}\right)$ then $\left(C, \sigma_{c}\right)$ is of the form " $1-1-\cdots-1-n_{1}-n_{1}-\cdots-n_{1}-\cdots-n_{p}-n_{p}-\cdots-n_{p}$ " where the number of l's appearing is one, if $F_{1}$ is a singleton, or more than one, otherwise.

The rest follows from Lemmas 2.16 and 2.18 and by noticing that the reduced \#-chain of $\left(C, \sigma_{c}\right)$ is either $\Gamma_{h}$ or $1+\cdots+\Gamma_{h}$.

Definition 5.14. A subcontinuum $H$ of a chainable continuum $M$ is an end-continuum of $M$ iff for every $\varepsilon>0$ there exists a chain-cover $C=\left\{C_{i} \mid i=1, \ldots, \mu\right\}$ of $M$ of mesh less than $\varepsilon$ such that $C_{1} \cap H \neq \varnothing$.

THEOREM 5.15. Let $M$ be a tree-like continuum and $h$ a divisible homeomorphism such that $M_{h}$ is chainable and $\pi\left(F_{1}\right)$ is an end-continuum of $M_{h}$. Then $M_{h}$ is perfect.

Proof. It follows from Lemma 5.12 and the proof of Theorem 5.13.

Notice that these results not only guarantee the existence of covers of $M_{h}$ of the right kind but assure it for any cover of sufficiently small mesh.

Theorem 5.15 suggests the following

Question 5.16. If $M$ is tree-like and $h$ is divisible and such that $M_{h}$ is chainable and $\pi\left(F_{1}\right)$ is an end-continuum, is $h$ perfect?

Once $M_{h}$ is perfect, it seems that $h$ can also be made perfect by first lifting every cover $C$ of $M_{h}$ according to its natural \#-graph $\sigma_{c}$ and then applying Theorem 5.13. However, things do not work so nicely as we can see from the following

ExAmple 5.17. Consider the continuum $M$ which is the intersection of covers following the pattern shown in Figure 5.2(a). Consider the period-2 homeomorphism with one fixed point $p$ induced by the period- 2 homeomorphisms shown at the bottom of the figure. It is not hard to see that this pair satisfies the hypothesis of Theorem 5.15. Therefore $M_{h}$ is perfect. 


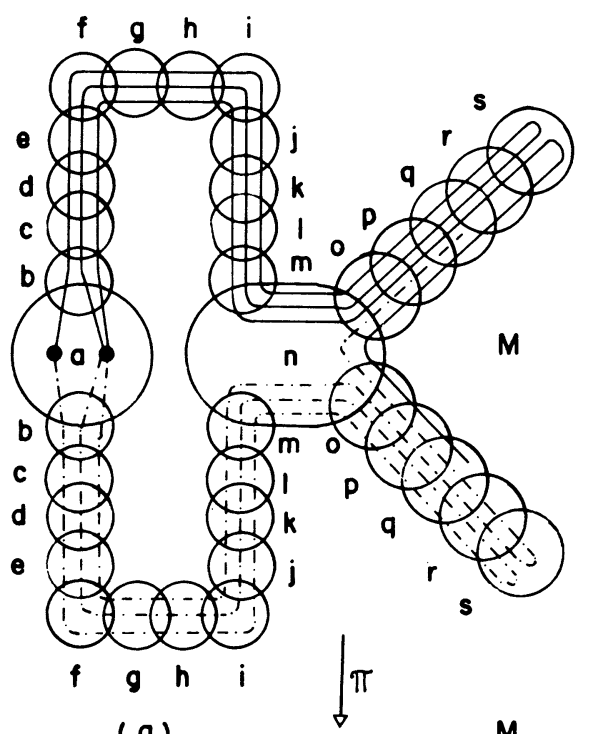

(a)

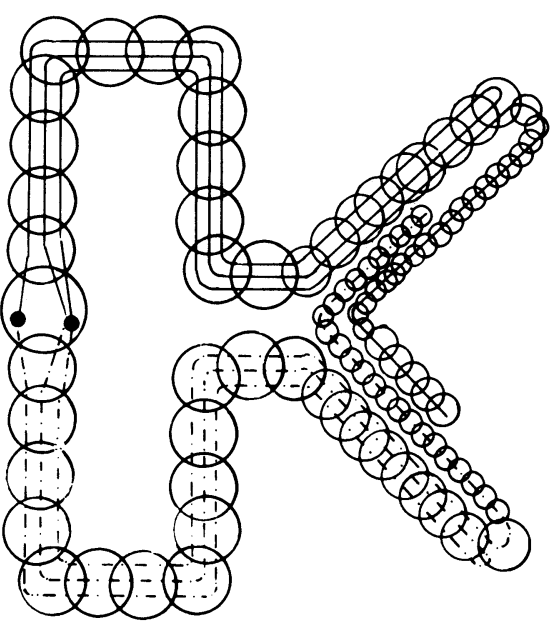

(b)

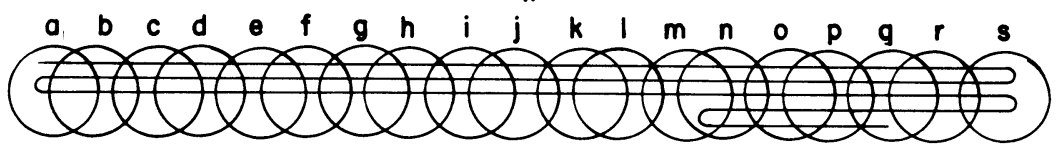

(c)

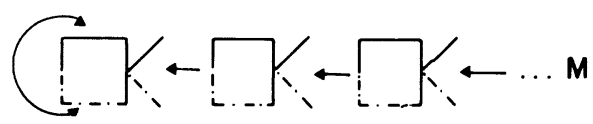

FIGURE 5.2

The chain-cover $C$ of $M_{h}$ shown in Figure 5.2(c) could be obtained by refining the projection $\pi(\mathbb{Q})$ of a spread cover $\mathbb{Q}$ of $M$, as shown in Figure 5.3. Observe then, that the coarser cover of $M$ of Figure 5.2(a) is just $L(C, \mathbb{Q})$, i.e., the lift of $C$ induced by Q.

The natural \#-graph of $C$ takes the value 2 for all elements of $C$ except for the link marked with " $a$ ", where it takes the value 1 . However, $C$ cannot be lifted properly since there is no possible way of 2-lifting the link marked with " $n$ ". This shows that it is important to choose the appropriate sequence of chain covers of $M_{h}$.

Despite this, $h$ is perfect as we can see from the consolidation shown in Figure 5.2(b).

Now, let us look at the modification of this example shown in Figure 5.4(a). The arrows point to the difference. In this case it is not obvious that $h$ is perfect since we cannot do a consolidation similar to that of Figure 5.2(b).

This new example is planar, atriodic and " $x$-odic" as shown in Figure 5.4(b), where a consolidation of the type of Figure 5.2(b) is presented.

It even seems possible that, by defining the embeddings more precisely, the example becomes nonchainable (by forcing it to have positive span [Le]). That would constitute a negative answer to Question 5.16. 


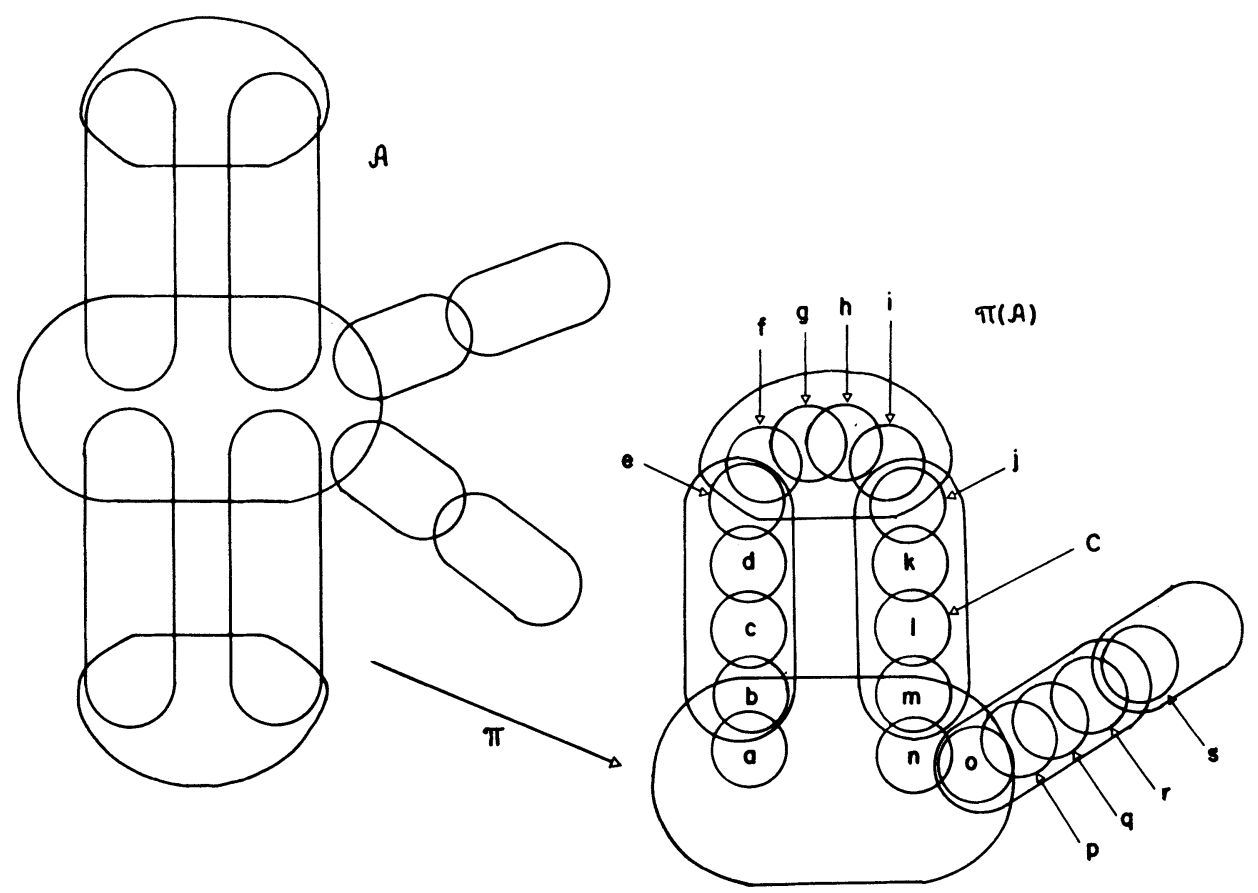

FiguRE 5.3

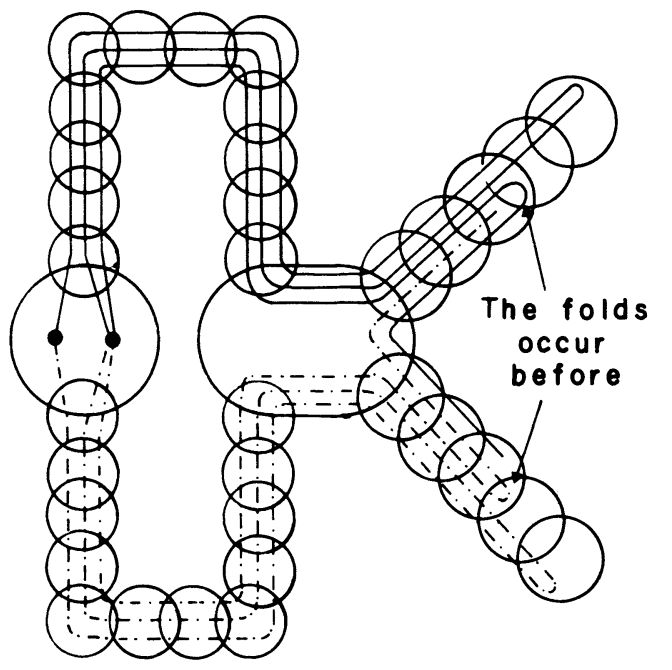

(a)

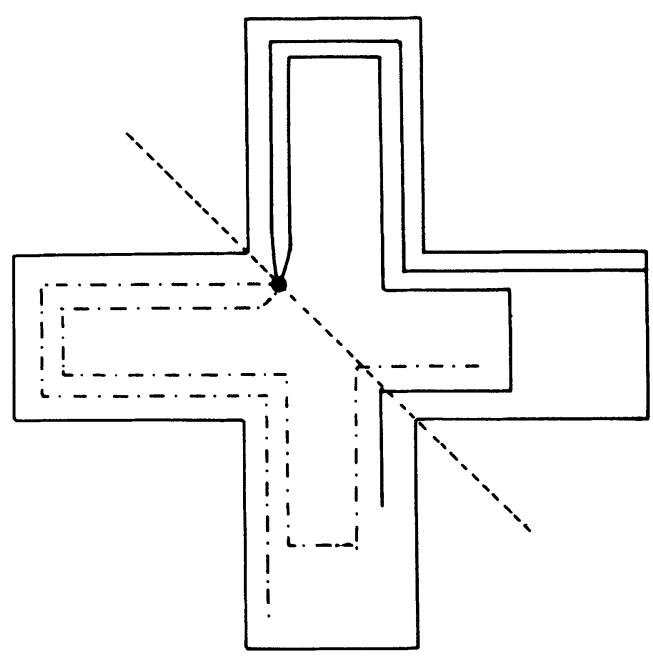

(b)

FIGURE 5.4 
In [T], several perfect homeomorphisms on chainable continua were constructed. It was even suggested [T] that there possibly exist perfect homeomorphisms of all possible kinds for the pseudo-arc.

REMARK 5.18. Due to the hereditary indecomposability of the pseudo-arc, every periodic homeomorphism on it is divisible. In addition, its orbit space is also a pseudo-arc $[\mathbf{R}]$ and therefore it is homogeneous. Then, any proper subcontinuum of it (the orbit space), in particular the projection of the fixed point set, is an end-continuum.

This remark and Theorem 5.15 tell us that the orbit spaces in the case of the pseudo-arc are always perfect.

Therefore, Question 5.16 can be particularized into

QUESTION 5.19 (BRECHNER). Is every periodic homeomorphism on the pseudo-arc perfect?

A positive answer to this question would represent tremendous progress in the study of the conjugacy classes of periodic homeomorphisms of the pseudo-arc.

And, of course, we can pose the question in its full generality:

QUESTION 5.20. Given any divisible homeomorphism of a tree-like continuum $M$, does the condition " $M_{h}$ is perfect" imply that $h$ is perfect?

We conclude this section with two interesting observations.

REMARK 5.21. Since the existence of a perfect period-2 homeomorphism on a continuum $M$ makes $M$ chainable, we have that no period-2 homeomorphism on a nonchainable continuum can be perfect.

REMARK 5.22. Any conjugate of a perfect homeomorphism is perfect.

6. Conjugacy. In this section we develop some tools that represent a useful aid in the study of the conjugacy classes of periodic homeomorphisms of tree-like continua and particularly of the pseudo-arc.

In the whole section, $h_{1}: M_{1} \rightarrow M_{1}$ and $h_{2}: M_{2} \rightarrow M_{2}$ will denote periodic homeomorphisms on the tree-like continua $M_{1}$ and $M_{2}$. The orbit spaces will be denoted by $M_{h_{1}}$ and $M_{h_{2}}$ and $\pi_{1}: M_{1} \rightarrow M_{h_{1}}$ and $\pi_{2}: M_{2} \rightarrow M_{h_{2}}$ will denote the projection maps.

DEFINITION 6.1. Let $f: M_{h_{1}} \rightarrow M_{h_{2}}$ be a map. We say that $f$ preserves multiplicity or is multiplicity preserving iff mult $f(x)=$ mult $x$ for all $x \in M_{h_{1}}$.

DEFINITION 6.2. Let $\mathscr{Q}^{1}$ and $\mathscr{Q}^{2}$ be finite open covers of $M_{1}$ and $M_{2}$, respectively. We say that $\mathscr{Q}^{1}$ and $\mathscr{Q}^{2}$ are $h_{1}-h_{2}$-isomorphic iff for $\lambda=1,2, \mathscr{O Q D}^{\lambda}$ is invariant under $h_{\lambda}$ and there exists a simplicial homeomorphism $\phi$ such that the diagram

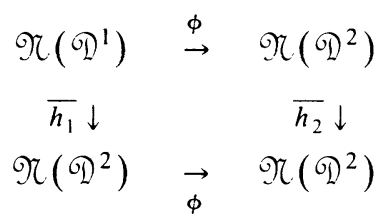

commutes, where $\overline{h_{\lambda}}$ is the simplicial homeomorphism induced by $h_{\lambda}, \lambda=1,2$. 
THEOREM 6.3. Let $M_{1}$ and $M_{2}$ be two tree-like continua and let $h_{1}: M_{1} \rightarrow M_{1}$ and $h_{2}$ : $M_{2} \rightarrow M_{2}$ be two divisible periodic homeomorphisms with orbit spaces $M_{h_{1}}$ and $M_{h_{2}}$. Suppose that there exists a multiplicity preserving homeomorphism $f: M_{h_{1}} \rightarrow M_{h_{2}}$. Then, given $\varepsilon>0$, there exist finite open covers $\mathrm{O}^{1}$ of $M_{1}$ and $\mathrm{Q}^{2}$ of $M_{2}$ of mesh less than $\varepsilon$ and such that ${ }^{10}{ }^{1}$ and ()$^{2}{ }^{2}$ are $h_{1}-h_{2}$-isomorphic.

Proof. During the proof, in each usage of the letter " $\lambda$ ", we will be assuming implicitly that the statement works for both $\lambda=1,2$. $[\mathbf{M}])$.

Let $\pi_{\lambda}: M_{\lambda} \rightarrow M_{h_{\lambda}}$ denote the projection maps. Since $M_{\lambda}$ is tree-like, so is $M_{h_{\lambda}}$ (see

Let $\mathbb{C}^{\prime \prime}=\left\{C^{1 i} \mid i=1, \ldots, \infty\right\}$ be a sequence of tree-covers of $M_{h_{1}}$ such that mesh $C^{1 i}<\varepsilon_{i}, \lim \varepsilon_{i}=0$ and $C^{1 i}=\left\{C_{j}^{1 i} \mid j=1, \ldots, l_{i}\right\}$. Then $\mathbb{C}^{2}=f\left(\mathrm{C}^{1}\right)=\left\{C^{2 i} \mid i=\right.$ $1, \ldots \infty\}$ where $C^{2 i}=\left\{C_{j}^{2 i}=f\left(C_{j}^{1 i}\right) \mid j=1, \ldots, l_{i}\right\}$ is a sequence of tree-covers of

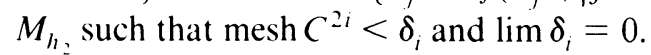

Using Theorem 4.13 find spread covers ( $^{\lambda}{ }^{\lambda}=\left\{T\left[a_{i}^{\lambda}\right] \mid i=1, \ldots, \alpha_{\lambda}\right\}$ of $M_{\lambda}$ of size less than $\varepsilon$. Then $\pi_{\lambda}\left(c^{\lambda \lambda}\right)$ is a finite open cover of $M_{h_{\lambda}}$. Use its Lebesgue number to find $\bar{k}>0$ such that

$$
C_{i}^{\lambda \bar{k}} \cap C_{j}^{\lambda \bar{k}} \neq \varnothing \text { implies } C_{i}^{\lambda \bar{k}} \cup C_{j}^{\lambda \bar{k}} \subset \pi_{\lambda}\left(T\left[a^{\lambda}\right]\right) \text { for some } a^{\lambda} \in I\left(\mathbb{Q}^{\lambda}\right)
$$

Let $W_{\lambda}=L\left(C^{\lambda \bar{k}}, \mathbb{Q}^{\lambda}\right)$ (see Definition 4.15). Use the Lebesgue number of $W_{\lambda}$ and Theorem 4.13 to find a spread cover $\mathscr{B}^{\lambda}=\left\{T\left[b_{i}^{\lambda}\right] \mid i=1, \ldots, \beta_{\lambda}\right\}$ of $M_{h}$ such that

$$
\operatorname{Shr} \mathscr{B}^{\lambda}<W_{\lambda}<\operatorname{Shr} \mathbb{Q}^{\lambda}
$$

Therefore $\pi_{\lambda}\left(\mathscr{B}^{\lambda}\right)<\pi_{\lambda}\left(W_{\lambda}\right)=C^{\lambda \bar{k}}$. Now use the Lebesgue number of $\pi_{\lambda}\left(\mathscr{B}^{\lambda}\right)$ to find $k>\bar{k}$ such that

$$
C^{\lambda k}<\pi_{\lambda}\left(\Re^{\lambda}\right)<C^{\lambda \bar{k}}
$$

We will proceed now to construct the cover $\mathscr{D}^{\lambda}$ of $M_{\lambda}$ by lifting the cover $C^{\lambda k}$ in the following way. As graphs, $C^{1 k}$ and $C^{2 k}$ are isomorphic. Let us define a $\#$-graph $\sigma: C^{\lambda k} \rightarrow N^{+}$by

$$
\sigma_{i}=\sigma\left(C_{i}^{\lambda k}\right)=\text { g.c.d. }\left(\text { ord } L\left(C_{i}^{1 k}, \mathscr{B}^{1}\right), \text { ord } L\left(C_{i}^{2 k}, \mathscr{乃}^{2}\right)\right)
$$

Since $h_{\lambda}$ is divisible, the range of $\sigma$ is a chain, and therefore $\left(C^{\lambda k}, \sigma\right)$ is a divisible \#-graph. 


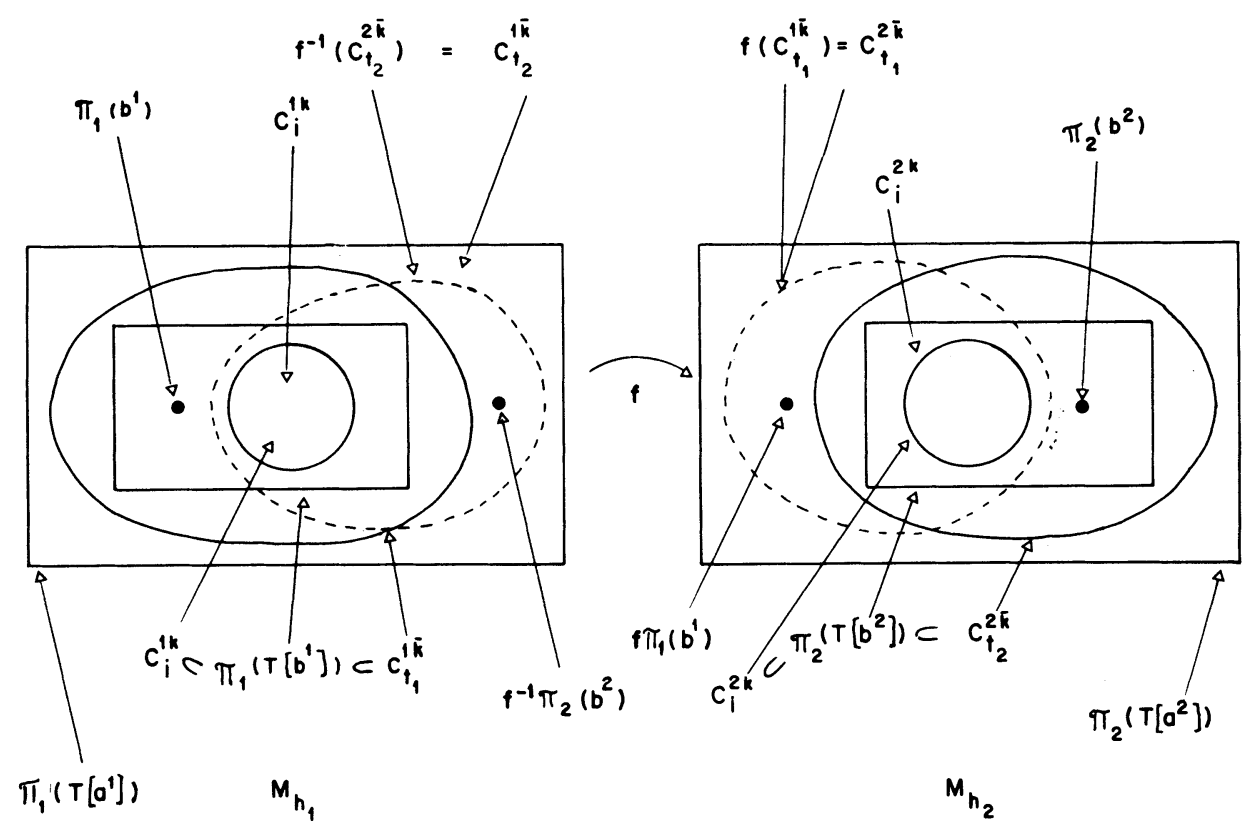

Figure 6.1

Now define $\mathscr{Q}^{\lambda}=L_{\sigma}\left(C^{\lambda k}, \mathscr{B}^{\lambda}\right)$. If $L_{\sigma_{i}}\left(C_{i}^{\lambda k}, \mathscr{B}^{\lambda}\right)=\left\{D_{i j}^{\lambda} \mid j=0, \ldots, \sigma_{i}-1\right\}$ then

$$
\mathscr{Q}^{\lambda}=\left\{D_{i j}^{\lambda} \mid i=1, \ldots, l_{k} ; j=0, \ldots, \sigma_{i}-1\right\} \text {. }
$$

By Theorem 4.24, $\mathfrak{X}\left(\mathscr{Q}^{\lambda}\right)$ is the $\left(C^{\lambda k}, \sigma\right)$-bellows. Hence by Remark $2.14, \mathscr{R}\left(\mathscr{Q}^{1}\right)$ and $\mathscr{N}\left(\mathscr{Q}^{2}\right)$ are isomorphic. Indeed, the function $\phi^{\prime}: \operatorname{vrt} \mathfrak{\Re}\left(\mathscr{Q}^{1}\right) \rightarrow \operatorname{vrt} \mathscr{N}\left(\mathscr{Q}^{2}\right)$ defined by $\phi^{\prime}\left(D_{i j}^{1}\right)=D_{i j}^{2}$ is a graph-isomorphism. Extend $\phi^{\prime}$ linearly to a simplicial homeomorphism $\phi: \Re\left(\mathscr{\mathscr { D }}^{1}\right) \rightarrow \Re\left(\mathscr{Q}^{2}\right)$.

Let $\bar{h}_{\lambda}: \mathscr{N}\left(\mathscr{Q}^{1}\right) \rightarrow \mathscr{N}\left(\mathscr{Q}^{1}\right)$ be the isomorphism induced by $h_{\lambda}$. Since

$$
\begin{aligned}
\bar{h}_{2} \phi\left(D_{i j}^{1}\right) & =\bar{h}_{2}\left(D_{i j}^{2}\right)=D_{i\left(j+1 \bmod \sigma_{i}\right)}^{2} \\
& =\phi\left(D_{i\left(j+1 \bmod \sigma_{i}\right)}^{1}\right)=\phi \bar{h}_{1}\left(D_{i j}^{1}\right),
\end{aligned}
$$

$\mathscr{Q}^{1}$ and $\mathscr{Q}^{2}$ are $h_{1}-h_{2}$-isomorphic.

The only thing left to prove is that mesh $\mathscr{O}^{\lambda}<\varepsilon$.

Fix $1 \leqslant i \leqslant l_{k}$ (see Figure 6.1). By (3), for some $t_{\lambda}$ with $1<t_{\lambda} \leqslant l_{k}$ and some $b^{\lambda} \in I\left(\mathscr{B}^{\lambda}\right)$,

$$
C_{i}^{\lambda k} \subset \pi_{\lambda}\left(T\left[b^{\lambda}\right]\right) \subset C_{t_{\lambda}}^{\lambda \bar{k}}
$$

where ord $b^{\lambda}=$ ord $L\left(C_{i}^{\lambda k}, \mathscr{B}^{\lambda}\right)$. Then

$$
C_{i}^{1 k}=f^{-1}\left(C_{i}^{2 k}\right), \quad f^{-1}\left(C_{t_{2}}^{2 \bar{k}}\right)=C_{t_{2}}^{1-\bar{k}}
$$

and

$$
C_{i}^{2 k}=f\left(C_{i}^{1 k}\right), \quad f\left(C_{t_{1}}^{1 \bar{k}}\right)=C_{t_{1}}^{2 \bar{k}}
$$


Thus, by (1), (4) and (5),

$$
C_{i}^{1 k} \subset C_{t_{1}}^{1 \bar{k}} \cup C_{t_{2}}^{1 \bar{k}} \subset \pi_{1}\left(T\left[a^{1}\right]\right)
$$

and

$$
C_{i}^{2 k} \subset C_{t_{1}}^{2 \bar{k}} \cup C_{t_{2}}^{2 \bar{k}} \subset \pi_{2}\left(T\left[a^{2}\right]\right)
$$

for some $a^{\lambda} \in I\left(Q^{\lambda}\right)$. Observe from (4) that $\pi_{1}\left(b^{1}\right) \in C_{t_{1}}^{1 \bar{k}}$ and $\pi_{2}\left(b^{2}\right) \in C_{t_{2}}^{2 \bar{k}}$ so $f^{-1} \pi_{2}\left(B^{2}\right) \in C_{t_{2}}^{1-k}$ and $f \pi_{1}\left(b^{1}\right) \in C_{t_{1}}^{2 \bar{k}}$. Therefore

$$
\begin{gathered}
\left\{\pi_{1}\left(b^{\mathrm{i}}\right), f^{-1} \pi_{2}\left(b^{2}\right)\right\} \subset \pi_{1}\left(T\left[a^{1}\right]\right), \\
\left\{\pi_{2}\left(b^{2}\right), f \pi_{1}\left(b^{1}\right)\right\} \subset \pi_{2}\left(T\left[a^{2}\right]\right),
\end{gathered}
$$

But

$$
\text { mult } f \pi_{1}\left(b^{1}\right)=\text { mult } \pi_{1}\left(b^{1}\right)=\text { ord } b^{1}=\text { ord } L\left(C_{i}^{1 k}, \Re^{1}\right)
$$

and

$$
\text { mult } f \pi_{2}\left(b^{2}\right)=\text { mult } \pi_{2}\left(b^{2}\right)=\text { ord } b^{2}=\text { ord } L\left(C_{i}^{2 k}, \Re^{2}\right)
$$

so by Lemma 3.9 ord $a^{\lambda} \mid \sigma_{i}=$ g.c.d.(ord $b^{1}$, ord $b^{2}$ ).

We have the following situation: $C_{i}^{\lambda k} \subset \pi_{\lambda}\left(T\left[b^{\lambda}\right]\right) \subset \pi_{\lambda}\left(T\left[a^{\lambda}\right]\right)$. The two latter sets lift to $T\left[b^{\lambda}\right]$ of order ord $b^{\lambda}$ and $T\left[a^{\lambda}\right]$ of order ord $a^{\lambda}$, respectively. Also $T\left[b^{\lambda}\right]<T\left[a^{\lambda}\right]$ following (2), and furthermore ord $a^{\lambda}\left|\sigma_{i}\right|$ ord $b^{\lambda}$. Then we can apply Lemma 3.14(iii) to obtain

$$
L_{\sigma i}\left(C_{i}^{\lambda k}, \mathscr{B}^{\lambda}\right)<L\left(C_{i}^{\lambda k}, \mathbb{Q}^{\lambda}\right)<\operatorname{Shr} \mathbb{Q}^{\lambda}
$$

Since size $Q<\varepsilon, \operatorname{mesh} L_{\sigma_{i}}\left(C_{i}^{\lambda k}, \Re^{\lambda}\right)<\varepsilon$. All we have to do now is to recall that $\mathscr{Q}^{\lambda}=L_{\sigma}\left(C^{\lambda k}, \mathscr{B}^{\lambda}\right)$ to have mesh $\mathscr{Q}^{\lambda}<\varepsilon$. This concludes the proof.

REMARK 6.4. Although the assumption of $h_{1}$ and $h_{2}$ being divisible was essential in the part of the proof where Theorem 4.24 was applied, it was not necessary in the proof of "mesh ${ }^{\lambda}{ }^{\lambda}<\varepsilon$ ".

Theorem 6.3 and Corollary 5.4 tell us that we can induce $h_{1}$ and $h_{2}$ using homeomorphic factor spaces $A_{i} \simeq B_{i}$ :

$$
\begin{array}{lccccccccc}
A_{1} & \stackrel{\alpha_{1}}{\leftarrow} & A_{2} & \stackrel{\alpha_{2}}{\leftarrow} & A_{3} & \leftarrow & \cdots & A & \stackrel{\alpha}{\rightarrow} & M_{1} \\
h_{1}^{1} \downarrow & & h_{1}^{2} \downarrow & & h_{1}^{3} \downarrow & & & \tilde{h}_{1} \downarrow & & \downarrow h_{1} \\
A_{1} & \stackrel{\alpha_{1}}{\leftarrow} & A_{2} & \underset{\alpha_{2}}{\leftarrow} & A_{3} & \leftarrow & \cdots & A & \stackrel{\alpha}{\rightarrow} & M_{1}
\end{array}
$$

and

$$
\begin{array}{lccccccccc}
B_{1} & \stackrel{\beta_{1}}{\leftarrow} & B_{2} & \stackrel{\beta_{2}}{\leftarrow} & B_{3} & \leftarrow & \ldots & B & \stackrel{\beta}{\rightarrow} & M_{2} \\
h_{2}^{1} \downarrow & & h_{2}^{2} \downarrow & & h_{2}^{3} \downarrow & & & \tilde{h}_{2} \downarrow & & \downarrow h_{2} \\
B_{1} & \leftarrow & B_{2} & \leftarrow & B_{3} & \leftarrow & \cdots & B & \stackrel{\beta}{\rightarrow} & M_{2}
\end{array}
$$


Furthermore, Theorem 6.3 says that there exist homeomorphisms $\phi_{i}$ making the diagrams

$$
\begin{array}{ccc}
A_{i} & \stackrel{\phi_{i}}{\rightarrow} & B_{i} \\
h_{1}^{i} \downarrow & & \downarrow h_{2}^{i} \\
A_{i} & \stackrel{\phi_{i}}{\rightarrow} & B_{i}
\end{array}
$$

commute.

At first glance one would be tempted to take the inverse limit of the $\phi_{i}$ 's and obtain a homeomorphism $\phi$ such that the following three-dimensional diagram

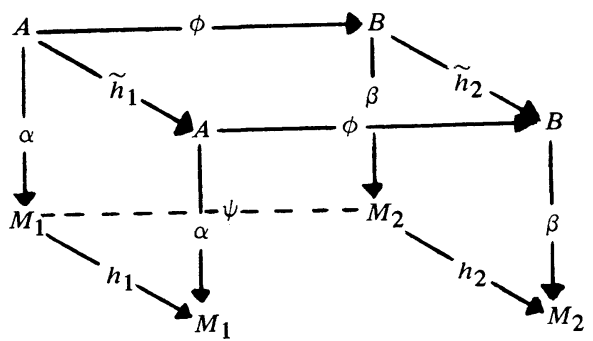

commutes. Then just define $\psi: M_{1} \rightarrow M_{2}$ by $\psi=\beta \phi \alpha^{-1}$ and observe that

$$
\begin{aligned}
\psi^{-1} h_{2} \psi & =\left(\beta \phi \alpha^{-1}\right)^{-1} h_{2}\left(\beta \phi \alpha^{-1}\right)=\alpha \phi^{-1}\left(\beta^{-1} h_{2} \beta\right) \phi \alpha^{-1} \\
& =\alpha\left(\phi^{-1} \tilde{h}_{2} \phi\right) \alpha^{-1}=\alpha \tilde{h}_{1} \alpha^{-1}=h_{1} .
\end{aligned}
$$

That is, $h_{1}$ and $h_{2}$ are conjugate.

Unfortunately, this is not always possible because in order to induce $\phi$ from the $\phi_{i}$ 's, we need

$$
\begin{array}{lccccccc}
A_{1} & \stackrel{\alpha_{1}}{\leftarrow} & A_{2} & \stackrel{\alpha_{2}}{\leftarrow} & A_{3} & \leftarrow & \ldots & A \\
\phi_{1} \downarrow & & \phi_{2} \downarrow & & \phi_{3} \downarrow & & & \downarrow \phi \\
B_{1} & \stackrel{\beta_{1}}{\leftarrow} & B_{2} & \stackrel{\beta_{2}}{\leftarrow} & B_{3} & \leftarrow & \cdots & B
\end{array}
$$

to commute. In general the bonding maps $\alpha_{i}$ and $\beta_{i}$ are different.

The next example shows a case where this diagram fails to commute.

EXAMPLE 6.5. In Figure 6.2 we display constructions for a case satisfying the hypothesis of Theorem 6.3.

In Figure 6.2(a) we have $M_{1}$, the 3-to-1 Knaster continuum, with a $180^{\circ}$ rotation as the period-2 homeomorphism $h_{1}$. Its orbit space is homeomorphic to $M_{1}$. 

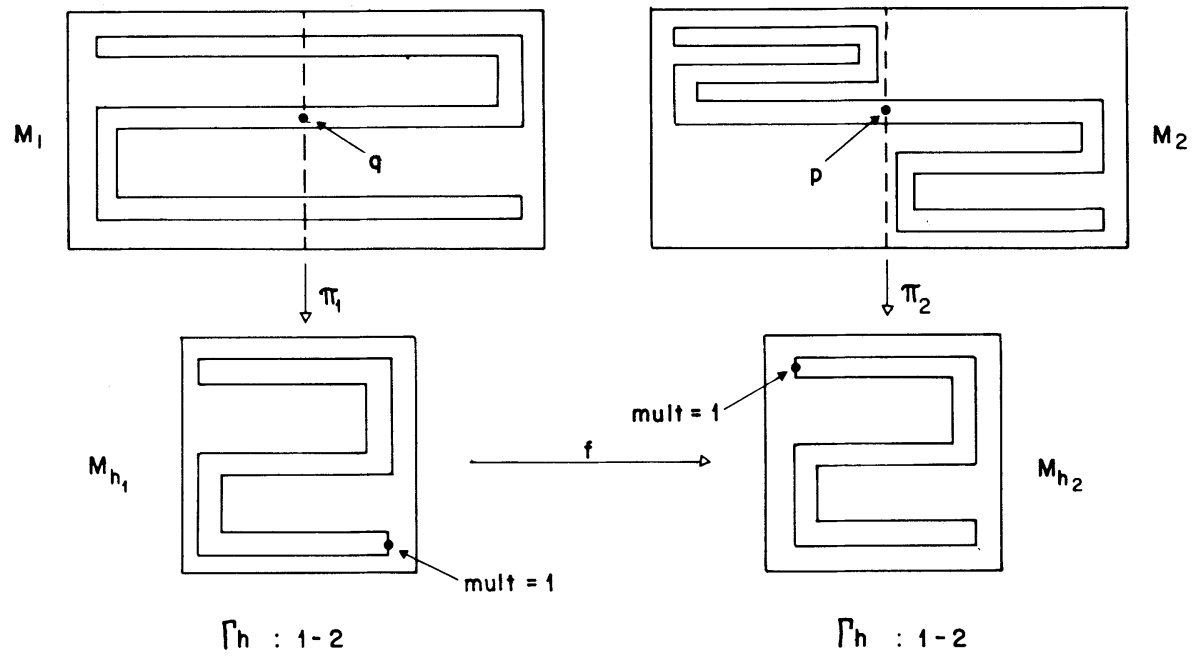

(a)

(b)

FIGURE 6.2

In Figure 6.2(b) we have $M_{2}$ defined as the intersection of the covers following the pattern shown. Again $h_{2}$ will be a $180^{\circ}$ rotation. $M_{h_{2}}$ is the 3-to-1 Knaster continuum and furthermore, there exists a multiplicity preserving homeomorphism $f: M_{h_{1}} \rightarrow M_{h_{2}}$.

Nevertheless, $h_{1}$ and $h_{2}$ are not conjugate since $M_{2}$ contains a cut-point $(p)$ and $M_{1}$ does not have any, so $M_{1}$ and $M_{2}$ can not be homeomorphic.

This example suggests the following

QuESTION 6.6. Under the hypothesis of Theorem 6.3, if $M_{1}$ is homeomorphic to $M_{2}$, are $h_{1}$ and $h_{2}$ conjugate?

Now let us consider the particular case of the pseudo-arc. In Remark 5.18, we saw that every periodic homeomorphism on the pseudo-arc is divisible. Also, since the pseudo-arc is hereditarily equivalent, every subcontinuum of it is either a point or a pseudo-arc.

REMARK 6.7. If $h$ is divisible with $\Gamma_{h}=\left\{1=n_{1}<n_{2}<\cdots<n_{p}\right\}$, then, since $F_{n_{p}}=M$, the period of $h$ must be $n_{p}$. Therefore the \#-graph of any period- $n$ homeomorphism on the pseudo-arc is of the form $\Gamma_{h}=\left\{1=n_{1}<n_{2}<\cdots<n_{p}=\right.$ $n\}$.

THEOREM 6.8. Let $h_{1}$ and $h_{2}$ be two period-n homeomorphisms on the pseudo-arc such that $\Gamma_{h_{1}}=\Gamma_{h_{2}}$. Let $F_{1}^{1}$ and $F_{1}^{2}$ be their respective fixed point sets. If either both $F_{1}^{1}$ and $F_{1}^{2}$ are degenerate or both are nondegenerate, then there exists a multiplicity preserving homeomorphism $f: M_{h_{1}} \rightarrow M_{h_{2}}$.

Proof. Since both $h_{1}$ and $h_{2}$ are divisible and $\Gamma_{h_{1}}=\Gamma_{h_{2}}=\Gamma$, the sets $\left\{\pi\left(F_{i}^{\lambda}\right) \mid i \in\right.$ $\Gamma\}$ are nested in $M_{h_{\lambda}}, \lambda=1,2$. That is, if $\Gamma=\left\{1=n_{1} \approx \cdots<n_{p}=n\right\}$ then $\pi\left(F_{1}^{\lambda}\right) \subset \pi\left(F_{2}^{\lambda}\right) \subset \cdots \subset \pi\left(F_{n}^{\lambda}\right)=M_{h_{\lambda}}$. 
Since either both $\pi\left(F_{1}^{1}\right)$ and $\pi\left(F_{1}^{2}\right)$ are degenerate or both are nondegenerate and $M_{h_{\lambda}}$ is a pseudo-arc, they are homeomorphic. Let $f_{1}: \pi\left(F_{1}^{1}\right) \rightarrow \pi\left(F_{1}^{2}\right)$ be a homeomorphism. Use Theorem 6 of $[\mathbf{L}]$ to extend $f_{1}$ to $f_{2}: \pi\left(F_{n_{2}}^{1}\right) \rightarrow \pi\left(F_{n_{2}}^{2}\right)$ and then $f_{2}$ to $f_{3}$ : $\pi\left(F_{n_{3}}^{1}\right) \rightarrow \pi\left(F_{n_{3}}^{2}\right)$ and so on.

At the end we will come up with the desired multiplicity preserving homeomor$\operatorname{phism} f=f_{p}: M_{h_{1}} \rightarrow M_{h_{2}}$.

It follows from this theorem that any two periodic homeomorphisms of the pseudo-arc with the same \#-graph and with homeomorphic fixed-point sets satisfy the hypothesis of Theorem 6.3. Then Question 6.6 can be particularized as follows.

QUESTION 6.9. If two periodic homeomorphisms of the pseudo-arc have the same \#-graph and homeomorphic fixed-point sets, are they conjugate?

A positive answer to either question will narrow the number of conjugacy classes of periodic homeomorphisms associated with a specific \#-graph to at most two classes.

\section{REFERENCES}

[A] R. Arens, Topologies for homeomorphism groups, Amer. J. Math. 68 (1964), 593-610.

[B] R. H. Bing, A homogeneous indecomposable plane continuum, Duke Math. J. 15 (1948), 729-742.

[Br] B. L. Brechner, Periodic homeomorphisms on chainable continua, Fund. Math. 64 (1969), 197-202.

[FM] J. B. Fugate and T. B. McLean, Compact groups of homeomorphisms on tree-like continua, Trans. Amer. Math. Soc. 267 (1981), 609-620.

[K] S. K. Kaul, Realization of mappings as inverse limits, Colloq. Math. 17 (1967), 297-307.

[L] G. R. Lehner, Extending homeomorphisms on the pseudo-arc, Trans. Amer. Math. Soc. 98 (1961), 369-394.

[Le] A. Lelek, Disjoint mappings and the span of spaces, Fund. Math. 55 (1964), 199-214.

[Lew] W. Lewis, Periodic homeomorphisms of chainable continua, preprint 1979.

[M] T. B. McLean, Confluent images of tree-like curves are tree-like, Duke Math. J. 39 (1972), 464-473.

[R] I. Rosenholtz, Open maps of chainable continua, Proc. Amer. Math. Soc. 42 (1974), 258-264.

[T] J. A. Toledo, More periodic homeomorphisms on chainable indecomposable continua, preprint, 1982.

Departamento de Matemáticas, Universidad autónoma Metropolitana-Iztapalapa, México D. F., MEXICO 